Geometry $\&$ Topology

Volume 6 (2002) 219-267

Published: 3 May 2002

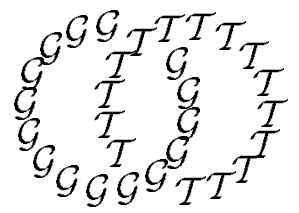

\title{
Deformation and rigidity of simplicial group actions on trees
}

\author{
Max Forester \\ Mathematics Institute, University of Warwick \\ Coventry, CV4 7AL, UK \\ Email: forester@maths.warwick.ac.uk
}

\begin{abstract}
We study a notion of deformation for simplicial trees with group actions ( $G-$ trees). Here $G$ is a fixed, arbitrary group. Two $G$-trees are related by a deformation if there is a finite sequence of collapse and expansion moves joining them. We show that this relation on the set of $G$-trees has several characterizations, in terms of dynamics, coarse geometry, and length functions. Next we study the deformation space of a fixed $G$-tree $X$. We show that if $X$ is "strongly slide-free" then it is the unique reduced tree in its deformation space.

These methods allow us to extend the rigidity theorem of Bass and Lubotzky to trees that are not locally finite. This yields a unique factorization theorem for certain graphs of groups. We apply the theory to generalized Baumslag-Solitar groups and show that many have canonical decompositions. We also prove a quasi-isometric rigidity theorem for strongly slide-free $G$-trees.
\end{abstract}

AMS Classification numbers Primary: 20E08

Secondary: $57 \mathrm{M} 07,20 \mathrm{~F} 65$

Keywords: $\quad G$-tree, graph of groups, folding, Baumslag-Solitar group, quasiisometry

Proposed: Walter Neumann

Seconded: Cameron Gordon, Wolfgang Metzler
Received: 21 June 2001

Revised: 21 March 2002 


\section{Introduction}

In this paper we study a notion of deformation for simplicial trees with group actions ( $G$-trees). Here $G$ is a fixed, arbitrary group, and we consider actions that do not invert edges. Such actions correspond to graph of groups decompositions of $G$, according to Bass-Serre theory [13].

Our notion of deformation is based on collapse moves in graphs of groups, in which an edge carrying an amalgamation of the form $A *_{C} C$ is collapsed to a vertex with group $A$. This operation simplifies the underlying graph without enlarging any vertex or edge groups. Collapse moves can be defined and performed directly on $G$-trees as well. An elementary deformation is a finite sequence of collapse moves and their inverses (called expansion moves). We are interested in knowing when two $G$-trees are related by a deformation, and in what can be said about them if they are. These two questions are addressed in the two main theorems of the paper.

In order to discuss the first result we recall some definitions. If $X$ is a $G$-tree then an elliptic subgroup is a subgroup $H \subseteq G$ that fixes a vertex of $X$. Thus, the elliptic subgroups are precisely the vertex stabilizers and their subgroups. The length function $\ell_{X}: G \rightarrow \mathbb{Z}$ assigns to $\gamma \in G$ the minimum displacement of any vertex under $\gamma$. Here we regard $G$-trees as metric spaces by assigning length one to every edge. A quasi-isometry is a map which preserves the large scale geometry of a metric space. The definition is fairly standard; see section 7 for details. In this section we also define coarse equivariance, a slight weakening of the property of equivariance. Collecting together Theorem 4.2, Corollary 4.3, and Theorem 7.3, we have the first main result.

Theorem 1.1 Let $G$ be a group and let $X$ and $Y$ be cocompact $G$-trees. The following conditions are equivalent.

(a) $X$ and $Y$ are related by an elementary deformation.

(b) $X$ and $Y$ have the same elliptic subgroups.

(c) There is a coarsely equivariant quasi-isometry $\phi: X \rightarrow Y$.

If all vertex stabilizers are finitely generated then we may also include:

(d) The length functions $\ell_{X}$ and $\ell_{Y}$ vanish on the same elements of $G$.

Note that condition (b) does not require $X$ and $Y$ to have the same vertex stabilizers. In fact, trees related by a deformation can easily have different 
vertex stabilizers. Condition (b) arises naturally in many situations, making the implication (b) $\Rightarrow$ (a) quite useful.

Regarding condition (d), it is well known that in most cases a $G$-tree is determined by its length function [7]. Our result shows that if one has partial knowledge of $\ell_{X}$ (namely, its vanishing set), then $X$ is partly determined, in an understandable way: up to deformation or up to quasi-isometry. The implication $(\mathrm{c}) \Rightarrow(\mathrm{a})$ is also interesting, as it transforms a coarse, approximate relationship between $G$-trees into a precise one.

One direct application of Theorem 1.1 is a proof of the conjecture of Herrlich stated in [10] (see Corollary 4.5). For other applications it is helpful to know more about condition (a). This is the subject of the second result. We fix a $G^{-}$ tree $X$ and consider the set of all trees related to $X$ by elementary deformations. This set is called the deformation space of $X$. We say that a $G$-tree is reduced if it admits no collapse moves. Every cocompact $G$-tree can be made reduced by performing collapse moves.

A $G$-tree is strongly slide-free if its stabilizers satisfy the following condition: for any edges $e$ and $f$ with common initial vertex $v$, if $G_{e} \subseteq G_{f}$ then there is an element $\gamma \in G$ fixing $v$ and taking $e$ to $f$. In terms of graphs of groups this means that for every vertex group $A$, if $C$ and $C^{\prime}$ are neighboring edge groups then no conjugate (in $A$ ) of $C$ is contained in $C^{\prime}$. Our second main result shows that strongly slide-free trees are locally rigid, from the point of view of deformations. (See Theorem 5.17 for a more comprehensive statement.)

Theorem 1.2 Let $X$ and $Y$ be cocompact $G$-trees that are related by an elementary deformation. If $X$ is strongly slide-free and $Y$ is reduced, then there is a unique $G$-isomorphism $X \rightarrow Y$.

This means that if a deformation space contains a strongly slide-free $G$-tree, then this tree is the unique reduced $G$-tree in the space. Hence, all trees in the deformation space reduce (by collapse moves) to the same $G$-tree. Combining Theorems 1.1 and 1.2 we obtain the following rigidity theorem.

Corollary 1.3 Let $X$ and $Y$ be cocompact $G$-trees with the same elliptic subgroups. If $X$ is strongly slide-free and $Y$ is reduced then there is a unique isomorphism of $G$-trees $X \rightarrow Y$.

This result provides an answer to the question of Bass and Lubotzky that is raised in [4]. It generalizes their Rigidity Theorem, which applies only to locally finite trees. A statement that more closely resembles theirs is given in 
Corollary 6.5. In fact this latter result, expressed in graph of groups language, has an interesting application. Recall that a group is unsplittable if it admits no nontrivial graph of groups decomposition. Then we have:

Corollary 1.4 Every group is the fundamental group of at most one strongly slide-free graph of unsplittable finitely generated groups, with finite underlying graph.

The phrase "at most one" is meant up to graph of groups isomorphism in the sense of [1]. This result is somewhat analogous to the classical theorem that states that every group has at most one free product decomposition (up to rearrangement of factors) into groups that are not themselves free products [11, Section 35].

Our results can be applied to the study of generalized Baumslag-Solitar trees, which are $G$-trees whose vertex and edge stabilizers are all infinite cyclic. The groups $G$ that arise are called generalized Baumslag-Solitar groups. They include the classical Baumslag-Solitar groups, torus knot groups, and finite index subgroups of these groups. They have the virtue that their elliptic subgroups are uniquely determined, independently of the tree (except in some degenerate cases). Thus for any such group $G$, there is a single deformation space of $G$-trees. If there is a strongly slide-free $G$-tree then it is canonical, by Theorem 1.2. A general statement for generalized Baumslag-Solitar trees is given in Corollary 6.10 .

A geometric application of Theorem 1.2 is obtained using condition (c) of Theorem 1.1. It is a quasi-isometric rigidity theorem for trees. This result complements the work of Mosher, Sageev, and Whyte [12], though there is no direct connection between their work and ours; they study quasi-isometries of groups, whereas here we work with a fixed group.

Corollary 1.5 Let $X$ and $Y$ be reduced cocompact $G$-trees with $X$ strongly slide-free. Given a coarsely equivariant quasi-isometry $\phi: X \rightarrow Y$, there is a unique equivariant isometry $X \rightarrow Y$, and it has finite distance from $\phi$.

Thus, strongly slide-free cocompact $G$-trees are quasi-isometrically rigid, in the equivariant sense. This means in particular that the local geometry of $X$ is completely determined by its equivariant large scale geometry.

Finally we mention that Theorems 1.1 and 1.2 can be used to obtain uniqueness results for various decompositions of groups, such as one-ended decompositions 
of accessible groups and JSJ decompositions. These applications are described in detail in [9].

Our approach to proving Theorem 1.1 is based on the method of folding of $G$-trees. This technique was used by Chiswell in his thesis (see [6]), and has also been developed by Stallings, Bestvina and Feighn, Dunwoody, and others $[14,5,8]$. In this paper, in order to understand the most general situation, we define and analyze folds performed at infinity, or parabolic folds.

During such a move, various rays with a common end become identified. The simplest example is given by the quotient map of a parabolic tree, in which edges or vertices are identified if they have the same relative distance from the fixed end. The result is a linear tree. More generally, fewer identifications may be made, so that the result is "thinner" parabolic tree, or similar operations may be performed inside a larger $G$-tree. Under suitable conditions, a morphism between $G$-trees can be factored as a finite composition of folds, multi-folds, and parabolic folds. We show that such a morphism can be constructed when two $G$-trees have the same elliptic subgroups. Then the various types of folds are shown to be elementary deformations, in this particular situation.

The proof of the Theorem 1.2 relies on the notion of a telescoping of a $G$-tree. A telescoping is a structure on a $G$-tree $X$ which remembers a second $G$-tree from which $X$ originated. We show that if one begins with a strongly slide-free $G$-tree, then this structure is preserved by elementary deformations, and so a ghost of the original tree is always present throughout any deformation. The theorem is proved by observing that any maximal sequence of collapse moves will then recover the original tree.

Acknowledgements I would like to thank Koji Fujiwara and Peter Scott for helpful conversations related to this work. I also thank David Epstein for his encouragement. This work was supported by EPSRC grant GR/N20867.

\section{Basic properties of $G$-trees}

A graph $A=(V(A), E(A))$ is a pair of sets together with a fixed point free involution $e \mapsto \bar{e}$ of $E(A)$ and maps $\partial_{0}, \partial_{1}: E(A) \rightarrow V(A)$ such that $\partial_{i}(\bar{e})=$ $\partial_{1-i}(e)$ for every $e \in E(A)$. Elements of $V(A)$ are called vertices and elements of $E(A)$ are called edges. The pair $\{e, \bar{e}\}$ is called a geometric edge. An edge $e$ for which $\partial_{0} e=\partial_{1} e$ is called a loop. For each vertex $v \in V(A)$ we set $E_{0}(v)=\left\{e \in E(A) \mid \partial_{0} e=v\right\}$. 
A graph of groups $\mathbf{A}=(A, \mathscr{A}, \alpha)$ consists of the following data: a connected graph $A$, groups $\mathscr{A}_{a}(a \in V(A))$ and $\mathscr{A}_{e}=\mathscr{A}_{\bar{e}}(e \in E(A))$, and injective homomorphisms $\alpha_{e}: \mathscr{A}_{e} \rightarrow \mathscr{A}_{\partial_{0} e}(e \in E(A))$. Given a vertex $a_{0} \in V(A)$ there is a fundamental group $\pi_{1}\left(\mathbf{A}, a_{0}\right)$ whose isomorphism type does not depend on $a_{0}$ (see [13, Chapter I, Section 5.1]).

A tree is a connected graph with no circuits: if $\left(e_{1}, \ldots, e_{n}\right)$ is a path with $e_{i+1} \neq \bar{e}_{i}$ for all $i$ (ie, a path without reversals), then $\partial_{0} e_{1} \neq \partial_{1} e_{n}$.

A ray is a semi-infinite path without reversals. An end of a tree $X$ is an equivalence class of rays, where two rays are considered equivalent if their intersection is a ray. The set of ends is called the boundary of the tree, denoted $\partial X$. A subtree contains an end if it contains a representative of that end.

An automorphism of a tree is an inversion if it maps $e$ to $\bar{e}$ for some edge $e$.

Definition 2.1 Let $G$ be a group. A $G$-tree is a tree $X$ together with an action of $G$ on $X$ by automorphisms, none of which are inversions. There is a well known correspondence between $G$-trees and graphs of groups having fundamental group $G$. A good reference for this material is [1] (see also [13]).

Definition 2.2 A $G$-tree is minimal if it contains no proper invariant subtree. Correspondingly, a graph of groups is minimal if there is no proper subgraph $A^{\prime} \subsetneq A$ which carries the fundamental group of $\mathbf{A}$. That is, the injective homomorphism $\pi_{1}\left(\left(\left.\mathbf{A}\right|_{A^{\prime}}\right), a_{0}\right) \rightarrow \pi_{1}\left(\mathbf{A}, a_{0}\right)$ of fundamental groups induced by the inclusion of $\left.\mathbf{A}\right|_{A^{\prime}}$ into $\mathbf{A}$ is not surjective for any $A^{\prime} \subsetneq A$.

If $A$ has finite diameter then this condition is equivalent to the following property: for every vertex $v \in V(A)$ of valence one, the image of the neighboring edge group is a proper subgroup of the vertex group $\mathscr{A}_{v}$ (see $[1,7.12]$ ).

Definition 2.3 A $G$-tree is reduced if, whenever $G_{e}=G_{\partial_{0} e}$ for an edge $e$ of the tree, $\partial_{0} e$ and $\partial_{1} e$ are in the same $G$-orbit. This occurs if and only if the tree admits no "collapse moves" (see 3.2 below). The corresponding notion for graphs of groups is: for every edge $e \in E(A)$, if $\alpha_{e}\left(\mathscr{A}_{e}\right)=\mathscr{A}_{\partial_{0} e}$ then $e$ is a loop.

This definition differs from the notion of "reduced" used in [5]. Note that if a $G$-tree (or a graph of groups) is reduced then it is minimal.

Every cocompact $G$-tree can be made reduced by performing collapse moves until none are available. These moves are described in the next section. The resulting $G$-tree will depend on the particular sequence of collapse moves chosen. Similarly, graphs of groups with finite underlying graphs can be made reduced. 
Definition 2.4 Let $X$ be a $G$-tree. An element $\gamma \in G$ is elliptic if it has a fixed point, and hyperbolic otherwise. We define the length function of $X$ by

$$
\ell_{X}(\gamma)=\min _{x \in V(X)} d(x, \gamma x)
$$

Thus, $\ell_{X}(\gamma)=0$ if and only if $\gamma$ is elliptic. If $\gamma$ is hyperbolic then the set

$$
L_{\gamma}=\left\{x \in X \mid d(x, \gamma x)=\ell_{X}(\gamma)\right\}
$$

is a $\gamma$-invariant linear subtree, called the axis of $\gamma$. The action of $\gamma$ on its axis is by a translation of amplitude $\ell_{X}(\gamma)$.

A minimal subtree is a nonempty $G$-invariant subtree which is minimal. If $G$ contains a hyperbolic element then there is a unique minimal subtree, equal to the union of the axes of hyperbolic elements (see [1, 7.5]). If $G$ contains no hyperbolic elements then any global fixed point is a minimal subtree. It is possible for a $G$-tree to have no minimal subtree.

A $G$-tree is elliptic if there is a global fixed point. It is parabolic if there is a fixed end, and some element of $G$ is hyperbolic. It follows that the minimal subtree is also parabolic, and has quotient graph equal to a closed circuit. A parabolic $G$-tree may have two fixed ends. In this case the minimal subtree is a linear tree acted on by translations. Otherwise the fixed end is unique. (Any $G$-tree with three fixed ends is elliptic.) See [7, Section 2] for a more complete discussion of these facts.

Remark 2.5 In a parabolic $G$-tree the set of elliptic elements is a subgroup, whereas this conclusion is false for $G$-trees in general (cf Lemma 2.8). To see this, let $\varepsilon$ be a fixed end. Every elliptic element fixes pointwise a ray tending to $\varepsilon$. Since any two such rays have nonempty intersection, any two elliptic elements have a common fixed point. Thus, their product is elliptic.

A subgroup $H$ of $G$ is elliptic if it fixes a vertex of $X$ (ie, if $X$ is an elliptic $H-$ tree). This property is stronger than requiring the elements of $H$ to be elliptic, though in many cases these two properties coincide (cf Proposition 2.6).

Notation If $\gamma \in G$ and $H \subseteq G$ is a subgroup, set $H^{\gamma}=\gamma H \gamma^{-1}$. Note that this yields the identity $\left(H^{\delta}\right)^{\gamma}=H^{(\gamma \delta)}$. If $x$ is a vertex or edge of a $G$-tree then the stabilizer of $x$ is $G_{x}=\{\gamma \in G \mid \gamma x=x\}$. All group actions are on the left, so that $G_{\gamma x}=\left(G_{x}\right)^{\gamma}$.

If $x$ and $y$ are vertices, edges, or ends of a tree, let $[x, y]$ denote the unique smallest subtree containing $x$ and $y$. It is an unoriented segment (including 
edges and their inverses), possibly with zero or infinite length. In the case where $y$ is an end, we will sometimes use the more suggestive notation $[x, y)$. Two edges $e$ and $f$ are coherently oriented if they are members of an oriented path without reversals. This occurs if and only if the segment $\left[\partial_{0} e, \partial_{0} f\right]$ contains exactly one of $e, f$.

Proposition 2.6 (Tits $[15,3.4]$ ) Let $X$ be a $G$-tree. If every element of $G$ has a fixed point in $X$ then either there is a global fixed point, or there is a unique end $\varepsilon \in \partial X$ which is fixed by $G$. In the latter case, if $\left(x_{1}, x_{2}, x_{3}, \ldots\right)$ is a sequence of vertices or edges tending monotonically to $\varepsilon$, then $G_{x_{i}} \subseteq G_{x_{i+1}}$ for all $i$, with strict inclusion for infinitely many $i$, and $G=\bigcup_{i \geqslant 0} G_{x_{i}}$.

Lemma 2.7 Let $X$ be a $G$-tree. An element $\gamma \in G$ is hyperbolic under any one of the following conditions:

(a) $d(x, \gamma x)$ is odd for some vertex $x$;

(b) for some vertex $x$ not fixed by $\gamma$, the edges of the path $[x, \gamma x]$ map injectively to the edges of $G \backslash X$;

(c) for some edge $e$ not fixed by $\gamma$, the edges $e$ and $\gamma e$ are coherently oriented.

Proof If $\gamma$ is not hyperbolic then it fixes some vertex $v \in V(X)$. To rule out cases (a) and (b), suppose that $x$ is any vertex not fixed by $\gamma$. The subtree $T$ spanned by $v, x$, and $\gamma x$ is equal to $[v, x] \cup[v, \gamma x]$, and $[v, x] \cap[v, \gamma x]$ meets $[x, \gamma x]$ in a single vertex, $w$. This vertex is fixed by $\gamma$ and so $\gamma([w, x])=$ $[w, \gamma x]$. As $[x, \gamma x]=[x, w] \cup[w, \gamma x]$, this path has even length and contains a pair of edges which are related by $\gamma$. These two edges have the same image in $G \backslash X$. This shows that neither (a) nor (b) can hold.

To rule out case (c) let $e$ be the edge, and look at the subtree spanned by $e, \gamma e$, and $v$. Then as $\gamma([v, e])=[v, \gamma e]$, the edges $e$ and $\gamma e$ are both oriented toward $v$, or both oriented away from $v$. Since any oriented path traversing $[e, \gamma e]$ travels first toward $v$ and then away from $v$, the two edges are incoherently oriented, violating (c).

Lemma 2.8 (Hyperbolic Segment Condition) Let $X$ be a $G$-tree and let $e$ and $f$ be edges such that $[e, f]=\left[\partial_{0} e, \partial_{0} f\right]$. Given $\gamma_{e} \in G_{\partial_{0} e}-G_{e}$ and $\gamma_{f} \in G_{\partial_{0} f}-G_{f}$, the product $\gamma_{e} \gamma_{f}$ is hyperbolic and its axis contains $[e, f]$.

Proof The element $\gamma_{e} \gamma_{f}$ takes $\gamma_{f}^{-1}(e)$ to $\gamma_{e}(e)$ and one easily checks that these two edges are coherently oriented. Then $\gamma_{e} \gamma_{f}$ is hyperbolic by Lemma 2.7(c). The subtree $\bigcup_{n \in \mathbb{Z}}\left(\gamma_{e} \gamma_{f}\right)^{n}\left[\gamma_{f}^{-1}(e), e\right]$ is a linear $\gamma_{e} \gamma_{f}$-invariant subtree, and must therefore be the axis. Now note that $[e, f] \subseteq\left[\gamma_{f}^{-1}(e), e\right]$. 


\section{Moves and factorizations}

In this section we discuss ways of modifying a $G$-tree to obtain another. We also discuss how to factor the more complicated moves as compositions of simpler moves, under certain conditions. The simplest of these moves will be called elementary moves. None of the moves affect the group $G$. In particular, a sequence of moves relating two graphs of groups will induce an isomorphism between their fundamental groups.

In all of the descriptions below, $X$ is a $G$-tree and $\mathbf{A}=(A, \mathscr{A}, \alpha)$ is the corresponding graph of groups.

Remark 3.1 All of the moves described here preserve ellipticity of elements of $G$. In many cases this can be seen by noting that the move defines an equivariant map from $X$ to the resulting tree. The case of an expansion move is discussed in 3.3 below. The only remaining case, the slide move, follows from the case of an expansion. As for hyperbolicity, we note that folds and multi-folds may change hyperbolic elements into elliptic elements.

3.2 Collapse moves Let $e \in E(X)$ be an edge such that $G_{e}=G_{\partial_{0} e}$, and whose endpoints are in different $G$-orbits. To perform a collapse move one simply collapses $e$ and all of its translates $\gamma e$ (for $\gamma \in G$ ) to vertices. That is, one deletes $e$ and identifies its endpoints to a single vertex, and does the same with translates. The image vertex of $\partial_{0} e$ and $\partial_{1} e$ will then have stabilizer $G_{\partial_{1} e}$.

The resulting graph $Y$ clearly admits a $G$-action. To see that it is a tree, let $\left(e_{1}, \ldots, e_{n}\right)$ be an oriented path in $Y$ without reversals. The corresponding sequence of edges in $X$ forms a disjoint union of oriented paths, each without reversals. The unique path in $X$ from $\partial_{0} e_{1}$ to $\partial_{1} e_{n}$ alternates between these paths and paths in $(G e \cup G \bar{e})$. In particular it is not contained in $(G e \cup G \bar{e})$, and so the endpoints $\partial_{0} e_{1}$ and $\partial_{1} e_{n}$ map to different vertices of $Y$. Thus, the original path in $Y$ is not a circuit.

To perform a collapse move in $\mathbf{A}$ one selects an edge $e \in E(A)$ which is not a loop, such that $\alpha_{e}: \mathscr{A}_{e} \rightarrow \mathscr{A}_{\partial_{0} e}$ is an isomorphism. Then one removes $e$ and $\partial_{0} e$, leaving $\partial_{1} e$. Every edge $f$ with initial vertex $\partial_{0} e$ is given the new initial vertex $\partial_{1} e$, and each inclusion $\alpha_{f}$ is replaced with $\alpha_{\bar{e}} \circ \alpha_{e}^{-1} \circ \alpha_{f}$.

A collapse move simplifies the underlying graph of $\mathbf{A}$ without increasing any of the labels $\mathscr{A}_{v}, \mathscr{A}_{e}$. Recall from Definition 2.3 that $\mathbf{A}$ is reduced if and only if no collapse moves can be performed. 
3.3 Expansion moves An expansion move is the reverse of a collapse move. To perform it one chooses a vertex $v \in V(X)$, a subgroup $H \subseteq G_{v}$, and a collection $S \subseteq E_{0}(v)$ of edges such that $G_{f} \subseteq H$ for every $f \in S$. One then adds a new edge $e$ with $\partial_{0} e=v$, and detaches the edges of $S$ from $v$ and re-defines $\partial_{0} f=\partial_{1} e$ for every $f \in S$. Finally, one performs this operation at each translate $\gamma v$ using the subgroup $H^{\gamma} \subseteq G_{\gamma v}$ and the edges $\gamma S \subseteq E_{0}(\gamma v)$.

After the move, the stabilizer $G_{v}$ is unchanged and the new edge $e$ has stabilizer $H \subseteq G_{v}$. The stabilizers $G_{f}$, for $f \in S$, are also unchanged. Thus the set of elliptic elements $\mathscr{E}=\bigcup_{v \in V(X)} G_{v}$ is the same before and after the move.

In $\mathbf{A}$, an expansion can be performed by choosing a vertex $v \in V(A)$, a subgroup $H \subseteq \mathscr{A}_{v}$, and a collection $S \subseteq E_{0}(v)$ of edges such that $\alpha_{f}\left(\mathscr{A}_{f}\right) \subseteq H$ for every $f \in S$. One then adds a new edge $e$ with $\partial_{0} e=v$ and sets $\mathscr{A}_{e}=\mathscr{A}_{\partial_{1} e}=H, \alpha_{e}=$ inclusion, and $\alpha_{\bar{e}}=\mathrm{id}$. Finally one detaches $f$ from $v$ for each $f \in S$ and re-defines $\partial_{0} f$ to be $\partial_{1} e$. The inclusions $\alpha_{f}: \mathscr{A}_{f} \rightarrow \mathscr{A}_{v}$ are unchanged except that they are now regarded as maps into $H$.

Definition 3.4 Collapse moves and expansion moves are called elementary moves. A move which factors as a finite composition of elementary moves is called an elementary deformation. Thus slides, subdivision, and the reverse of subdivision are all elementary deformations (see 3.6, 3.7 below).

Remark 3.5 We have just seen that collapse and expansion moves preserve ellipticity and hyperbolicity of elements of $G$. In fact a slightly stronger statement holds: these moves do not change the set of elliptic subgroups of $G$. Recall that a subgroup $H \subseteq G$ is elliptic if and only if it is contained in a vertex stabilizer. During an elementary move there are two stabilizers involved (up to conjugacy), and the larger of these two is present before and after the move. Thus, if $H$ is contained in a stabilizer before the move, it is still contained in a stabilizer afterward.

3.6 Slide moves Suppose $e, f \in E(X)$ are adjacent edges (ie, $\partial_{0} e=\partial_{0} f$ ) such that $G_{f} \subseteq G_{e}$ and $f \notin G e \cup G \bar{e}$. To perform a slide move of $f$ over $e$, detach $f$ from $\partial_{0} e$ and re-define $\partial_{0} f$ to be $\partial_{1} e$; also do the same for all pairs $\gamma e, \gamma f$. The requirement that $G_{f} \subseteq G_{e}$ ensures that the equivariant move is well defined, and that $G$ still acts on the resulting tree.

To perform a slide move in $\mathbf{A}$ one selects adjacent edges $e, f \in E(A)$ such that $\alpha_{f}\left(\mathscr{A}_{f}\right) \subseteq \alpha_{e}\left(\mathscr{A}_{e}\right)$ and $f \neq e, \bar{e}$. Again one detaches $f$ from $\partial_{0} e$ and re-defines $\partial_{0} f$ to be $\partial_{1} e$. The inclusion map $\alpha_{f}$ is then replaced by $\alpha_{\bar{e}} \circ \alpha_{e}^{-1} \circ \alpha_{f}$. 
A slide move is equal to the composition of an expansion and a collapse. To see this, consider a slide move (in A) of $f$ over $e$. We have that $\alpha_{f}\left(\mathscr{A}_{f}\right) \subseteq$ $\alpha_{e}\left(\mathscr{A}_{e}\right) \subseteq \mathscr{A}_{\partial_{0} e}$ and $f \neq e, \bar{e}$. Expand at $\partial_{0} e$ using the subgroup $\mathscr{A}_{e} \subseteq \mathscr{A}_{\partial_{0} e}$, pulling across $e$ and $f$ to the new vertex. Then collapse $e$. The newly created edge takes the place of $e$ (and has label $\mathscr{A}_{e}$ ) and $f$ has been slid across it.

3.7 Subdivision This move is straightforward: simply insert a vertex in the interior of an edge $e$ and do the same for each of the translates $\gamma e$. The new vertex will have stabilizer $G_{e}$, as will the two "halves" of $e$.

In $\mathbf{A}$, insert a vertex in the interior of some $e \in E(A)$, and give it the label $\mathscr{A}_{e}$. Also give the two adjacent "half-edges" the label $\mathscr{A}_{e}$ and let the new inclusion maps be the identity. Note that a subdivision is a special case of an expansion move (just collapse either of the new half-edges to undo).

3.8 Folds To perform a fold one chooses edges $e, f \in E(X)$ with $\partial_{0} e=\partial_{0} f$ and identifies $e$ and $f$ to a single edge ( $\partial_{1} e$ and $\partial_{1} f$ are also identified). One also identifies $\gamma e$ with $\gamma f$ for every $\gamma \in G$, so the resulting graph is an equivariant quotient space of $X$. It is not difficult to show that the image graph is a tree.

The effect of a fold on the quotient graph of groups can vary, depending on how the edges and vertices involved meet the various $G$-orbits. The possibilities are discussed in some detail in [5]. To summarize, the fold is of type B if $e$ or $f$ projects to a loop in $G \backslash X$, and type A otherwise; and it is of type I, II, or III, accordingly as $V(G \backslash X)$ and $E(G \backslash X)$ both decrease, both remain unchanged, or only $E(G \backslash X)$ decreases.

A type B fold is equal to the composition of a subdivision, two type A folds, and the reverse of a subdivision. Thus in many situations it suffices to consider only type A folds.

3.9 Multi-folds This move is similar to a fold except that several edges are folded together at once. To perform a multi-fold, one chooses a vertex $v \in V(X)$ and a subset $S \subseteq E_{0}(v)$, and identifies all the edges of $S$ to a single edge. One also does the same for all sets of edges $\gamma S \subseteq E_{0}(\gamma v)$ for $\gamma \in G$, so the move is equivariant. Multi-folds are classified into types $\mathrm{A}$ and $\mathrm{B}$, and into types I, II, and III in the same way that folds are.

If $G \backslash X$ is finite then every multi-fold is a finite composition of type I and type III folds and type II multi-folds. 
In A, a type II multi-fold corresponds to "pulling a subgroup across an edge," described briefly in [5]. If the subgroup is finitely generated then the type II multi-fold is a finite composition of type II folds.

3.10 Parabolic folds This move may also be viewed as infinitely many folds (or multi-folds) performed in one step. It is similar to a type II multi-fold except that it is performed at an end $\varepsilon \in \partial X$ rather than at a vertex. During the move, various rays with end $\varepsilon$ are identified with each other. The exact definition is fairly technical so we begin with two examples.

3.10.A Example Let $G$ be the Baumslag-Solitar group $B S(1,6)$ with presentation $\left\langle x, t \mid t x t^{-1}=x^{6}\right\rangle$. This group admits a graph of groups decomposition in which the graph is a single loop, the vertex and edge groups are both $\mathbb{Z}$, and the inclusion maps are the identity and multiplication by 6 . The BassSerre tree $X$ has vertex stabilizers equal to the conjugates of the infinite cyclic subgroup $\langle x\rangle$. Note that as the tree is parabolic, the set of elliptic elements $\mathscr{E}=\bigcup_{i \in \mathbb{Z}}\left\langle t^{i} x t^{-i}\right\rangle$ is a subgroup.

Now let $Y$ be the linear $\mathbb{Z}$-tree where a generator of $\mathbb{Z}$ acts by a translation of amplitude 1 . There is a surjective homomorphism $B S(1,6) \rightarrow \mathbb{Z}$ defined by sending $x$ to 0 and $t$ to 1 . This homomorphism, with kernel $\mathscr{E}$, defines an action of $B S(1,6)$ on $Y$ in which every vertex and edge stabilizer is $\mathscr{E}$. Thus $X$ and $Y$ have the same elliptic and hyperbolic elements (as $B S(1,6)$-trees).

As a group, $\mathscr{E}$ is isomorphic to the additive subgroup $\mathbb{Z}[1 / 6] \subseteq \mathbb{Q}$ generated by integral powers of 6 . The quotient graph of groups of $Y$ has a loop as its underlying graph, and its vertex and edge groups are both $\mathbb{Z}[1 / 6]$. The inclusion maps are the identity and multiplication by 6 (which is an isomorphism).

The tree $Y$ is simply the quotient $G$-tree $\mathscr{E} \backslash X$. This transition from $X$ to $Y$, which is a special case of a parabolic fold, can also be achieved with an infinite sequence of type II folds which "zip" the parabolic tree down to a line.

3.10.B Example Again consider $B S(1,6)$ and its Bass-Serre tree $X$. Let $H$ be the subgroup $\mathbb{Z}[1 / 3] \subseteq \mathbb{Z}[1 / 6]=\mathscr{E}$. Let $e \in E(X)$ be an edge with $G_{e}=\mathbb{Z} \subseteq \mathbb{Z}[1 / 6]$, so that $G_{e} \subseteq H \subseteq \mathscr{E}$. Form a quotient space $Y$ of $X$ by identifying the orbit $H e$ to a single edge, and by extending these identifications equivariantly. Thus the orbits $\left(H^{\gamma}\right) \gamma e$ become edges, for each $\gamma \in G$.

The image of $e$ in $Y$ has stabilizer equal to $H$, and the quotient graph is again a loop. The quotient graph of groups has vertex and edge groups equal to $\mathbb{Z}[1 / 3]$ and the inclusion maps are the identity and multiplication by 6 . The tree $Y$ is the regular tree of valence three (cf Remark 3.14). 
3.10.C Definition Now we discuss the parabolic fold move. Let $e \in E(X)$ be an edge with $G_{e}=G_{\partial_{0} e}$ such that $\partial_{1} e=t \partial_{0} e$ for some $t \in G$. Let $\varepsilon$ be the end represented by the ray $\left(e, t e, t^{2} e, \cdots\right)$. Note that $t$ is hyperbolic (by Lemma 2.7(a)) and it fixes $\varepsilon$. Let $T$ be the connected component of the orbit $G\{e, \bar{e}\}$ that contains $e$, and let $G_{T}$ be the stabilizer of $T$. Note that $\gamma \in G_{T}$ if and only if $[e, \gamma e] \subseteq T$. In particular $G_{\varepsilon} \subseteq G_{T}$, since if $\gamma \in G_{\varepsilon}$ then $[e, \gamma e]$ is contained in $[e, \varepsilon) \cup[\gamma e, \varepsilon) \subseteq T$.

We claim that $G_{T}$ fixes $\varepsilon$. To see this, suppose that $\gamma \in G_{T}$ does not fix $\varepsilon$. Then there is a linear subtree $(\varepsilon, \gamma \varepsilon) \subseteq T$. Note that $t^{i} e \in(\varepsilon, \gamma \varepsilon)$ for sufficiently large $i$ and $\partial_{0} t^{i} e$ separates $t^{i} e$ from $\gamma \varepsilon$. Similarly, for large $i, \gamma t^{i} e \in(\varepsilon, \gamma \varepsilon)$ and $\partial_{0} \gamma t^{i} e$ separates $\gamma t^{i} e$ from $\varepsilon$. These observations imply that there is a vertex $v \in(\varepsilon, \gamma \varepsilon)$ such that both edges of $E_{0}(v) \cap(\varepsilon, \gamma \varepsilon)$ are in $G e$ (rather than $G \bar{e})$. Then there is an element $\delta \in G$ taking one of these edges to the other and fixing their initial endpoint $v$. A conjugate of $\delta$ fixes $\partial_{0} e$ without fixing $e$, contradicting the assumption that $G_{e}=G_{\partial_{0} e}$.

Thus $G_{T} \subseteq G_{\varepsilon}$ and so $T$ is a parabolic $G_{T}$-tree. In fact we now have that $G_{T}=G_{\varepsilon}$. Note that $\varepsilon$ is the unique fixed end of $T$ that is separated from $e$ by $\partial_{1} e$. Therefore $\varepsilon$ is well defined without reference to $t$. Now let $\mathscr{E} \subseteq G$ be the set of elliptic elements.

Choose a subgroup $H \subseteq\left(G_{\varepsilon} \cap \mathscr{E}\right)$ with $G_{e} \subseteq H$. Based on $H$ and $e$, we will define an equivalence relation on $X$ whose quotient space is a $G$-tree. This tree will be the "largest" quotient $G$-tree with the property that the stabilizer of the image of $e$ contains $H$. The element $t$ is used in the definition, but the move is independent of the choice of $t$.

Let $\Sigma$ be the semigroup $\left\{t^{-i} \mid i \geqslant 0\right\}$. We define a relation on $E(X)$ as follows: set $e \approx h^{\sigma} e$ for every $h \in H$ and $\sigma \in \Sigma$, and extend equivariantly: $\gamma e \approx \gamma h^{\sigma} e$ for every $\gamma \in G, h \in H$, and $\sigma \in \Sigma$. This relation is reflexive and symmetric, but we must pass to its transitive closure to obtain an equivalence relation. It will be helpful to express this transitive closure explicitly. Note that $\gamma e \approx \gamma h_{1}^{\sigma_{1}} e \approx \gamma h_{1}^{\sigma_{1}} h_{2}^{\sigma_{2}} e \approx \cdots \approx \gamma h_{1}^{\sigma_{1}} \cdots h_{j}^{\sigma_{j}} e$, so set

$$
\gamma e \sim \gamma h_{1}^{\sigma_{1}} \cdots h_{j}^{\sigma_{j}} e
$$

where $\gamma \in G, h_{i} \in H$, and $\sigma_{i} \in \Sigma$ for each $i$. Let $\left\langle H^{\Sigma}\right\rangle$ denote the subgroup of $G$ generated by the elements $h^{\sigma}(h \in H, \sigma \in \Sigma)$. The relation $\sim$ is symmetric and reflexive, and clearly $e_{0} \approx e_{1}$ implies $e_{0} \sim e_{1}$. For transitivity, suppose that $e_{0} \sim e_{1}$ and $e_{1} \sim e_{2}$. We can write $e_{0}=\gamma e$ and $e_{1}=\gamma h_{1}^{\sigma_{1}} \cdots h_{j}^{\sigma_{j}} e$, and also $e_{1}=\delta e$ and $e_{2}=\delta k_{1}^{\tau_{1}} \cdots k_{l}^{\tau_{l}} e$. Then $e=\delta^{-1} \gamma h_{1}^{\sigma_{1}} \cdots h_{j}^{\sigma_{j}} e$. This shows 
that $\delta^{-1} \gamma \in G_{e}\left\langle H^{\Sigma}\right\rangle=\left\langle H^{\Sigma}\right\rangle$. Here we use the assumption that $G_{e} \subseteq H$. We now have

$$
\begin{aligned}
e_{2} & =\delta k_{1}^{\tau_{1}} \cdots k_{l}^{\tau_{l}} \delta^{-1} \gamma h_{1}^{\sigma_{1}} \cdots h_{j}^{\sigma_{j}} e \\
& =\gamma\left(\gamma^{-1} \delta\right) k_{1}^{\tau_{1}} \cdots k_{l}^{\tau_{l}}\left(\delta^{-1} \gamma\right) h_{1}^{\sigma_{1}} \cdots h_{j}^{\sigma_{j}} e,
\end{aligned}
$$

showing that $e_{0} \sim e_{2}$, as $\left(\gamma^{-1} \delta\right),\left(\delta^{-1} \gamma\right) \in\left\langle H^{\Sigma}\right\rangle$. Thus, $\sim$ is the transitive closure of $\approx$.

Next we verify that $\sim$ is independent of the choice of $t$. Suppose that $\partial_{1} e=$ $s \partial_{0} e$ for some $s \in G$. Then $s^{-1} t \partial_{0} e=\partial_{0} e$ and so $s^{-1} t \in G_{\partial_{0} e}=G_{e} \subseteq H$. Let $k=s^{-1} t$. Notice that for any $h \in H$ and $i \geqslant 0$,

$$
\begin{aligned}
h^{t^{-i}} & =\left(k^{-1} s^{-1}\right) \cdots\left(k^{-1} s^{-1}\right) h(s k) \cdots(s k) \\
& =k^{-1}\left(k^{-1}\right)^{s^{-1}}\left(k^{-1}\right)^{s^{-2}} \cdots\left(k^{-1}\right)^{s^{-(i-1)}} h^{s^{-i}} k^{s^{-(i-1)}} \cdots k^{s^{-1}} k .
\end{aligned}
$$

Thus $e_{0} \approx_{t} e_{1}$ implies $e_{0} \sim_{s} e_{1}$, where the subscripts indicate whether $t$ or $s$ has been used. Similarly $e_{0} \approx_{s} e_{1}$ implies $e_{0} \sim_{t} e_{1}$, and therefore $\sim_{s}$ is the same relation as $\sim_{t}$. Furthermore, the subgroup $\left\langle H^{\Sigma}\right\rangle$ is independent of the choice of $t$.

Now let $Y$ be the quotient $G$-graph $X / \sim$. The quotient map $X \rightarrow Y$ is called a parabolic fold. We claim that $Y$ is a tree. First consider the quotient $T / \sim$. We begin by observing that every ray of the form $[x, \varepsilon) \subseteq T$ maps injectively to $T / \sim$. Indeed, if two vertices $u, v \in[x, \varepsilon)$ are related by $\sim$ then there is an element $\gamma \in\left(G_{\varepsilon} \cap \mathscr{E}\right)$ taking $u$ to $v$. Then $\gamma([u, \varepsilon))=[v, \varepsilon)$, and one of these rays contains the other. Applying Lemma 2.7(c) to an edge of $[u, \varepsilon)$ one finds that $\gamma$ is hyperbolic, unless $u=v$. The latter must occur since $\gamma \in \mathscr{E}$.

Now suppose that $e_{0} \sim f_{0}$ where $e_{0}, f_{0} \in E(T)$. To show that $T / \sim$ is a tree it suffices to show that $\left[e_{0}, \varepsilon\right)$ and $\left[f_{0}, \varepsilon\right)$ have the same image, so that the image of their union is a ray in $T / \sim$. Write $\left[e_{0}, \varepsilon\right)=\left(e_{0}, e_{1}, e_{2}, \ldots\right)$ and $\left[f_{0}, \varepsilon\right)=\left(f_{0}, f_{1}, f_{2}, \ldots\right)$ (these are oriented paths without reversals). We need to show that $e_{i} \sim f_{i}$ for each $i$, given that $e_{0} \sim f_{0}$. So suppose that $e_{0}=\gamma e$ and $f_{0}=\gamma h_{1}^{\sigma_{1}} \cdots h_{j}^{\sigma_{j}} e$. Note that $[e, \varepsilon)=\left(e, t e, t^{2} e, \ldots\right)$, and so $e_{i}=\gamma t^{i} e$ for each $i$. Then $f_{0}=\gamma h_{1}^{\sigma_{1}} \cdots h_{j}^{\sigma_{j}} \gamma^{-1} e_{0}$, and also $\gamma h_{1}^{\sigma_{1}} \cdots h_{j}^{\sigma_{j}} \gamma^{-1}$ fixes $\varepsilon$. Thus $\gamma h_{1}^{\sigma_{1}} \cdots h_{j}^{\sigma_{j}} \gamma^{-1}\left(\left[e_{0}, \varepsilon\right)\right)=\left[f_{0}, \varepsilon\right)$ and so $f_{i}=\gamma h_{1}^{\sigma_{1}} \cdots h_{j}^{\sigma_{j}} \gamma^{-1} e_{i}$ for each $i$. We now have

$$
\begin{aligned}
f_{i} & =\gamma h_{1}^{\sigma_{1}} \cdots h_{j}^{\sigma_{j}} \gamma^{-1} e_{i} \\
& =\left(\gamma t^{i}\right) t^{-i} h_{1}^{\sigma_{1}} \cdots h_{j}^{\sigma_{j}} t^{i} e \\
& =\left(\gamma t^{i}\right) h_{1}^{\left(t^{-i} \sigma_{1}\right)} \cdots h_{j}^{\left(t^{-i} \sigma_{j}\right)} e .
\end{aligned}
$$


Note that $t^{-i} \sigma_{l} \in \Sigma$ for each $l \leqslant j$ because $i \geqslant 0$. Thus, writing $f_{i}$ as above and $e_{i}$ as $\left(\gamma t^{i}\right) e$, we see that $e_{i} \sim f_{i}$. Therefore $T / \sim$ is a tree. It follows that $Y$ is also a tree because all identifications occur in $T$ and its translates. Line (3.12) demonstrates the need for involving the semigroup $\Sigma$ in the definition, since the exponents $t^{-i}$ are unavoidable if the quotient space is to be a tree.

Next we consider stabilizers in $Y$. These are simply the stabilizers of the equivalence classes in $X$. We have

$$
\begin{aligned}
{[e] } & =\left\{\gamma h_{1}^{\sigma_{1}} \cdots h_{j}^{\sigma_{j}} e \mid \gamma \in G_{e}, h_{i} \in H, \sigma_{i} \in \Sigma, j \geqslant 0\right\} \\
& =\left\{h_{1}^{\sigma_{1}} \cdots h_{j}^{\sigma_{j}} e \mid h_{i} \in H, \sigma_{i} \in \Sigma, j \geqslant 0\right\} \quad \text { as } G_{e} \subseteq H
\end{aligned}
$$

and hence $G_{[e]}=\left\langle H^{\Sigma}\right\rangle$. Similarly $G_{\left[\partial_{0} e\right]}=\left\langle H^{\Sigma}\right\rangle$, and the stabilizers of translates of $[e]$ and $\left[\partial_{0} e\right]$ are obtained by conjugation. If $x \in\left(X-\left(G e \cup G \bar{e} \cup G \partial_{0} e\right)\right)$ then $[x]=\{x\}$ and $G_{[x]}=G_{x}$. Notice that the union of all stabilizers is unchanged, as $\left\langle H^{\Sigma}\right\rangle \subseteq\left(G_{\varepsilon} \cap \mathscr{E}\right)$. Therefore parabolic folds preserve ellipticity and hyperbolicity of elements of $G$.

One last observation is that the quotient graph does not change during a parabolic fold; each equivalence class $[x]$ is contained in the orbit $G x$.

To summarize:

Proposition 3.13 Let $e$ be an edge of a $G$-tree such that $G_{e}=G_{\partial_{0} e}$ and $\partial_{1} e=t \partial_{0} e$ for some $t \in G$. Suppose a parabolic fold is performed at e using the subgroup $H$, according to relation (3.11). Then the quotient space is a $G$-tree and the images of $e$ and $\partial_{0} e$ have stabilizers $\left\langle H^{\Sigma}\right\rangle$. Outside of $G e \cup G \bar{e} \cup G \partial_{0} e$ the tree and its stabilizer data are unchanged. The move preserves ellipticity and hyperbolicity of elements of $G$, and induces an isomorphism of quotient graphs.

Remark 3.14 In Examples 3.10.A and 3.10.B the tree $Y$ cannot be obtained from $X$ by an elementary deformation. One way to see this is to note that the trees have different modular homomorphisms, whereas elementary moves preserve this invariant. The modular homomorphism $q: G \rightarrow \mathbb{Q}^{\times}$of a locally finite $G$-tree is defined by

$$
q(\gamma)=\left[V: V \cap V^{\gamma}\right] /\left[V^{\gamma}: V \cap V^{\gamma}\right],
$$

where $V$ is any subgroup of $G$ commensurable with a vertex stabilizer. In this definition we are using the fact that in locally finite $G$-trees, vertex stabilizers are commensurable with all of their conjugates. One can easily check that $q$ 
is independent of the choice of $V$. Equivalent definitions and properties of the modular homomorphism are given in [3].

Recall that during an expansion or collapse move there is a vertex stabilizer that remains unchanged. Taking $V$ to be this stabilizer, one obtains invariance of $q$ under elementary moves.

In the two examples of 3.10, the image of the modular homomorphism of $X$ is generated by the element 6 . In Example 3.10.A, the tree $Y$ has trivial modular homomorphism. In Example 3.10.B the image of the modular homomorphism of $Y$ is generated by the element $[\mathbb{Z}[1 / 3]: 6 \mathbb{Z}[1 / 3]]=2$. Evidently the modular homomorphism is not invariant under parabolic folding, or under infinite compositions of elementary moves.

Proposition 3.15 Let $\rho: X \rightarrow Y$ be a parabolic fold, performed at $e \in$ $E(X)$. Then $\rho$ is a finite composition of multi-folds if and only if $G_{\rho(e)}$ fixes a vertex of $X$. When this occurs, the multi-folds are of type II.

Proposition 3.15 will be proved in section 4, using the Multi-fold Lemma (4.8).

Proposition 3.16 If a fold or a type II multi-fold preserves hyperbolicity of elements of $G$ then it is a finite composition of elementary moves.

Proof First we show that the fold must be of type I or type II. Suppose that edges $e$ and $f$ generate the fold (so $\partial_{0} e=\partial_{0} f$ ) and that it is of type III. This means that $f \notin G e$ but $\partial_{1} f \in G \partial_{1} e$. Then $\partial_{1} f=\gamma \partial_{1} e$ for some $\gamma \in G$. If $f \notin G \bar{e}$ then $\gamma$ is hyperbolic by Lemma 2.7(b). Otherwise, if $f=\delta \bar{e}$ for some $\delta \in G$, then $\delta \partial_{1} e=\partial_{0} f$ and $\delta \partial_{0} f=\partial_{1} f$. Again by Lemma 2.7(b), $\delta$ is hyperbolic. We now re-define $\gamma$ to be $\delta^{2}$, which is hyperbolic and takes $\partial_{1} e$ to $\partial_{1} f$. In both cases, after the fold, the image vertex of $\partial_{1} e$ is fixed by $\gamma$. Hence $\gamma$ becomes elliptic, a contradiction.

Thus we consider type I folds and type II multi-folds. We can assume that the folds are of type A, as remarked in 3.8. Consider first a type I fold. In this situation, $f \notin(G e \cup G \bar{e})$ and $\partial_{1} f \notin\left(G \partial_{1} e \cup G \partial_{0} e\right)$. Since the stabilizer of the image of $\partial_{1} e$ is $\left\langle G_{\partial_{1} e}, G_{\partial_{1} f}\right\rangle$, this subgroup contains no hyperbolic elements. Thus it fixes an end or vertex $w \in X \cup \partial X$ by Proposition 2.6. Consider the paths $\left[w, \partial_{1} e\right]$ and $\left[w, \partial_{1} f\right]$. If $e \in\left[w, \partial_{1} e\right]$ then $G_{\partial_{1} e}=G_{e}$ as $G_{\partial_{1} e}$ fixes $w$. If in addition $f \notin\left[w, \partial_{1} f\right]$ then $f \in\left[w, \partial_{1} e\right]$, and so $G_{\partial_{1} e} \subseteq G_{f}$. The same result as the fold can now be achieved by sliding $e$ over $f$ and then collapsing $e$.

Similarly, if $f \in\left[w, \partial_{1} f\right]$ and $e \notin\left[w, \partial_{1} e\right]$ then the fold is equivalent to sliding $f$ over $e$ and then collapsing $f$. 
The last possibility is that $e \in\left[w, \partial_{1} e\right]$ and $f \in\left[w, \partial_{1} f\right]$ (the three cases correspond to whether the path from $w$ to $[e, f]$ joins at $\partial_{1} f$, at $\partial_{1} e$, or at $\left.\partial_{0} e=\partial_{0} f\right)$. Then $G_{e}=G_{\partial_{1} e}$ and $G_{f}=G_{\partial_{1} f}$ as before. In this case we collapse both $e$ and $f$ end expand a new edge with stabilizer $\left\langle G_{e}, G_{f}\right\rangle$, bringing across all edges which were previously incident to $\partial_{1} e$ or $\partial_{1} f$. The result is the same as the fold.

Finally, consider a type II multi-fold, performed at the set of edges $S \subseteq E_{0}(v)$. In this situation $S$ is contained in a single edge orbit $G e$, with $e \in S$. Consider $G_{e}$ and $G_{\partial_{1} e}$. Assuming that $|S|>1$ (otherwise the multi-fold is trivial) there is an edge $\gamma e \in S$ with $\gamma \in\left(G_{v}-G_{e}\right)$. The image vertex of $\partial_{1} e$ and $\partial_{1} \gamma e$ has stabilizer containing $G_{\partial_{1} e}$ and $G_{\partial_{1} \gamma e}$, and therefore $\left\langle G_{\partial_{1} e}, G_{\partial_{1} \gamma e}\right\rangle$ contains only elliptic elements. From the Hyperbolic Segment Condition (2.8) it follows that $G_{\partial_{1} e}=G_{e}$ (and $G_{\partial_{1} \gamma e}=G_{\gamma e}$ ). The multi-fold can be replaced by the following: collapse $e$ (the rest of $S$ collapses with it, by equivariance) and then expand a new edge with the appropriate stabilizer.

\section{Deformation of $G$-trees}

In this section we prove several of the implications comprising Theorem 1.1.

Definition 4.1 A group $G$ has property (FA) if every $G$-tree is elliptic. This notion is due to Serre. It is unsplittable if, in every $G$-tree, every element of $G$ is elliptic. Being unsplittable is equivalent to the property that $G$ admits no nontrivial graph of groups decomposition.

These properties often agree, but they do not agree in general. We say that $G$ has property (E) if there is a global fixed point in every $G$-tree in which the elements of $G$ are all elliptic. Thus, a group has property (FA) if and only if it is unsplittable and has property (E). Note that all finitely generated groups have property (E), by Proposition 2.6.

Theorem 4.2 Let $G$ be a group, and let $X$ and $Y$ be cocompact $G$-trees. Then $X$ and $Y$ are related by an elementary deformation if and only if they have the same elliptic subgroups.

Corollary 4.3 Let $G$ be a group. Let $X$ and $Y$ be cocompact $G$-trees whose vertex stabilizers have property (E). The following conditions are equivalent.

(a) $X$ and $Y$ are related by an elementary deformation. 
(b) $X$ and $Y$ define the same partition of $G$ into elliptic and hyperbolic elements.

(c) The length functions $\ell_{X}$ and $\ell_{Y}$ vanish on the same elements of $G$.

Proof The implications $(\mathrm{b}) \Leftrightarrow(\mathrm{c})$ are trivial, and $(\mathrm{a}) \Rightarrow(\mathrm{b})$ follows from Theorem 4.2. For $(\mathrm{b}) \Rightarrow(\mathrm{a})$, consider a stabilizer $G_{x}$ of some vertex $x \in V(X)$. As a group acting on $Y$ it consists of elliptic elements, and by property (E) it has a fixed point. Thus every $X$-elliptic subgroup is $Y$-elliptic, and conversely by symmetry. Now Theorem 4.2 yields conclusion (a).

Remark 4.4 In Corollary 4.3, the assumption of (E) vertex stabilizers is essential. The implication (b), (c) $\Rightarrow(\mathrm{a})$ can fail to hold for trees related by a parabolic fold, as shown in Remark 3.14.

The following result is the conjecture of Herrlich, slightly modified, from [10].

Corollary 4.5 Let A and B be graphs of groups having finite underlying graphs, whose vertex groups have property (FA). If $\mathbf{A}$ and $\mathbf{B}$ have isomorphic fundamental groups then there exist a graph of groups $\mathbf{B}^{\prime}$, an isomorphism of graphs of groups $\Phi: \mathbf{B}^{\prime} \rightarrow \mathbf{B}$, and a finite sequence of elementary moves taking A to $\mathbf{B}^{\prime}$.

The isomorphism of graphs of groups is meant in the sense of [1, Section 2]. This notion of isomorphism is more general than the naive notion (consisting of group isomorphisms satisfying the appropriate commutative diagrams). For example, without changing the isomorphism type, one can replace an inclusion map $\alpha_{e}: \mathscr{A}_{e} \rightarrow \mathscr{A}_{\partial_{0} e}$ by ad $(g) \circ \alpha_{e}: \mathscr{A}_{e} \rightarrow \mathscr{A}_{\partial_{0} e}$, for any $g \in \mathscr{A}_{\partial_{0} e}$. Here, $\operatorname{ad}(g): \mathscr{A}_{\partial_{0} e} \rightarrow \mathscr{A}_{\partial_{0} e}$ is defined by $\operatorname{ad}(g)(s)=g s g^{-1}$.

Proof Write $\mathbf{A}=(A, \mathscr{A}, \alpha)$ and $\mathbf{B}=(B, \mathscr{B}, \beta)$. Choose basepoints $a_{0} \in A$ and $b_{0} \in B$, and let $\psi: \pi_{1}\left(\mathbf{A}, a_{0}\right) \rightarrow \pi_{1}\left(\mathbf{B}, b_{0}\right)$ be an isomorphism. Setting $G=\pi_{1}\left(\mathbf{A}, a_{0}\right)$, the trees $X=\left(\widetilde{\mathbf{A}, a_{0}}\right)$ and $Y=\left(\widetilde{\mathbf{B}, b_{0}}\right)$ are $G$-trees (via $\psi$ in the case of $Y$ ). The vertex stabilizers of both trees have property (FA), so each stabilizer has fixed points in both trees. Hence $X$ and $Y$ have the same elliptic subgroups. Theorem 4.2 now provides a sequence of elementary moves from $X$ to $Y$. There is a corresponding sequence of elementary moves of graphs of groups taking $\mathbf{A}$ to a quotient graph of groups $\mathbf{B}^{\prime}$ of $Y$. Now, since $\mathbf{B}^{\prime}$ and $\mathbf{B}$ have $G$-isomorphic covering trees, there is an isomorphism $\Phi: \mathbf{B}^{\prime} \rightarrow \mathbf{B}$ by $[1$, $4.2-4.5]$. 
Next we turn to the proof of the Theorem 4.2, which occupies the rest of this section.

Definition 4.6 A map between trees (or graphs) is a morphism if it sends vertices to vertices and edges to edges (and respects the maps $\partial_{0}, \partial_{1}, e \mapsto \bar{e}$ ). Geometrically, it is a simplicial map which does not send any edge into a vertex.

Proposition 4.7 Let $G$ be a group and suppose that $\phi: X \rightarrow Y$ is an equivariant morphism of $G$-trees. Assume further that $\phi$ is surjective and $G \backslash X$ is finite. Then there exist a $G$-tree $Z$ and equivariant simplicial maps $X \stackrel{\rho}{\rightarrow} Z \stackrel{\psi}{\rightarrow} Y$ with $\phi=\psi \rho$, such that $\rho$ is a finite composition of folds and $\psi$ induces an isomorphism of quotient graphs.

This result is proved in [5, Proposition, Section 2]. The hypotheses are slightly different but their proof still works. The quotient graph $G \backslash Y$ is finite by equivariance and surjectivity, rather than by finite generation and minimality.

Lemma 4.8 (Multi-fold Lemma) Let $\phi: X \rightarrow Y$ be an equivariant morphism of $G$-trees. If $T \subseteq X$ is a simplicial subtree of finite diameter such that $\phi(T)$ is finite, then $\phi$ factors as $X \stackrel{\rho}{\rightarrow} X^{\prime} \stackrel{\phi^{\prime}}{\rightarrow} Y$ where $\rho$ is a finite composition of multi-folds and $\left.\phi^{\prime}\right|_{\rho(T)}$ is an embedding.

Proof To simplify the argument we use non-equivariant folds. We will prove that a factorization $\phi=\phi^{\prime} \rho$ exists, where $\rho$ is a finite composition of nonequivariant multi-folds and $\left.\phi^{\prime}\right|_{\rho(T)}$ is an embedding. If one then replaces the multi-folds of $\rho$ by their equivariant counterparts, the factorization is still valid.

We begin by defining an equivalence relation $\sim_{\phi}$ on the set $E(T) \times E(T)$. Choose a basepoint $v \in V(T)$ and let $\left[e, e^{\prime}\right]_{v}$ denote the subtree spanned by $e, e^{\prime}$, and $v$ (for $e, e^{\prime} \in E(T)$ ). Set $\left(e, e^{\prime}\right) \sim_{\phi}\left(f, f^{\prime}\right)$ if there exists a graph isomorphism $\left[e, e^{\prime}\right]_{v} \rightarrow\left[f, f^{\prime}\right]_{v}$ fixing $v$ and sending $e$ to $f$ and $e^{\prime}$ to $f^{\prime}$, such that the diagram

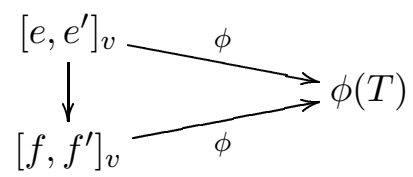

commutes. We will call the equivalence classes $(T, v, \phi)$-types. Since the tripods $\left[e, e^{\prime}\right]_{v}$ have bounded diameter and $\phi(T)$ is finite, there are only finitely many $(T, v, \phi)$-types. 
A subtree $S \subseteq T$ supports a $(T, v, \phi)$-type if the $(T, v, \phi)$-type contains a pair $\left(e, e^{\prime}\right)$ such that $\left[e, e^{\prime}\right]_{v} \subseteq S$. By taking a union of such tripods one can find a finite subtree $S$ which supports all $(T, v, \phi)$-types. For such an $S$ we claim that if $\left.\phi\right|_{S}$ is an embedding, then $\left.\phi\right|_{T}$ is an embedding. For otherwise, if $e \neq e^{\prime}$ and $\phi(e)=\phi\left(e^{\prime}\right)$ for some pair of edges $e, e^{\prime} \in E(T)$, then the $(T, v, \phi)$-type containing $\left(e, e^{\prime}\right)$ is not supported by $S$ (as $\left.\phi\right|_{\left[e, e^{\prime}\right]_{v}}$ is not injective).

A full multi-fold along $e$ is the multi-fold defined by identifying all of the edges $E_{0}\left(\partial_{0} e\right) \cap \phi^{-1} \phi(e)$ to one edge. It is the "maximal" multi-fold along $e$ through which $\phi$ factors.

Now let $S \subseteq T$ be a finite tree which supports all $(T, v, \phi)$-types, and suppose that $\left.\phi\right|_{S}$ is not an embedding. Then there are two vertices $u, w \in V(S)$ with the same image in $Y$. Since $Y$ contains no circuits, the image of the path from $u$ to $w$ must contain a reversal. Thus there exists a pair of edges $e_{0}, e_{0}^{\prime} \in E(S)$ such that $\partial_{0} e_{0}=\partial_{0} e_{0}^{\prime}$ and $\phi\left(e_{0}\right)=\phi\left(e_{0}^{\prime}\right)$. Among all such pairs choose $e_{0}$ and $e_{0}^{\prime}$ to minimize the distance $d$ from $\partial_{0} e_{0}$ to $v$. Factor $\phi$ as $\phi=\phi^{\prime} \rho$ where $\rho$ is the full multi-fold along $e_{0}$. We claim that $\rho$ preserves the equivalence relation, ie, that $\left(e, e^{\prime}\right) \sim_{\phi}\left(f, f^{\prime}\right)$ implies $\left(\rho(e), \rho\left(e^{\prime}\right)\right) \sim_{\phi^{\prime}}\left(\rho(f), \rho\left(f^{\prime}\right)\right)$.

To show this, let $T_{d} \subseteq T$ be the subtree spanned by the vertices having distance at most $d$ from $v$, and define $S_{d} \subseteq S$ similarly (note, $S_{d} \subseteq T_{d}$ ). By the choice of $e_{0},\left.\phi\right|_{S_{d}}$ is an embedding. In fact, since $S$ supports all $(T, v, \phi)$-types, $\left.\phi\right|_{T_{d}}$ is an embedding, and then we must have $S_{d}=T_{d}$. This last statement holds because if there were an edge $e \in E\left(T_{d}\right)-E\left(S_{d}\right)$ then by injectivity of $\left.\phi\right|_{T_{d}}$, the $(T, v, \phi)$-type containing $(e, e)$ would not be supported by $S$.

Now suppose that $\left(e, e^{\prime}\right) \sim_{\phi}\left(f, f^{\prime}\right)$ and consider the effect of $\rho$ on the tripods $\left[e, e^{\prime}\right]_{v}$ and $\left[f, f^{\prime}\right]_{v}$. Let $\alpha:\left[e, e^{\prime}\right]_{v} \rightarrow\left[f, f^{\prime}\right]_{v}$ be the graph isomorphism shown in diagram (4.9). Note that since $\left.\phi\right|_{T_{d}}$ is injective, the restriction $\left.\alpha\right|_{\left[e, e^{\prime}\right]_{v} \cap T_{d}}$ is the identity (and $\left[e, e^{\prime}\right]_{v} \cap T_{d}=\left[f, f^{\prime}\right]_{v} \cap T_{d}$ ). If $\partial_{0} e_{0} \notin\left[e, e^{\prime}\right]_{v}$ then $\partial_{0} e_{0} \notin\left[f, f^{\prime}\right]_{v}$ and $\rho$ is injective on both trees (here we use non-equivariance of $\rho$ ). Therefore $\left(\rho(e), \rho\left(e^{\prime}\right)\right) \sim_{\phi^{\prime}}\left(\rho(f), \rho\left(f^{\prime}\right)\right)$ via the isomorphism

$$
\left(\left.\rho\right|_{\left[f, f^{\prime}\right]_{v}}\right) \alpha\left(\left.\rho\right|_{\left[e, e^{\prime}\right]_{v}}\right)^{-1}:\left[\rho(e), \rho\left(e^{\prime}\right)\right]_{\rho(v)} \rightarrow\left[\rho(f), \rho\left(f^{\prime}\right)\right]_{\rho(v)}
$$

(cf diagram 4.10 below). If $\partial_{0} e_{0} \in\left[e, e^{\prime}\right]_{v}$ then $\partial_{0} e_{0} \in\left[f, f^{\prime}\right]_{v}$ and $\alpha$ maps $\left[e, e^{\prime}\right]_{v} \cap\left(E_{0}\left(\partial_{0} e_{0}\right) \cap \phi^{-1} \phi\left(e_{0}\right)\right)$ bijectively to $\left[f, f^{\prime}\right]_{v} \cap\left(E_{0}\left(\partial_{0} e_{0}\right) \cap \phi^{-1} \phi\left(e_{0}\right)\right)$. Note that $\rho$ identifies $E_{0}\left(\partial_{0} e_{0}\right) \cap \phi^{-1} \phi\left(e_{0}\right)$ to a single edge and is bijective on other geometric edges. Hence two edges of $\left[e, e^{\prime}\right]_{v}$ are identified by $\rho$ if and only if their images under $\alpha$ are identified by $\rho$. This implies that there is a 
graph isomorphism $\alpha^{\prime}$ making the following diagram commute:

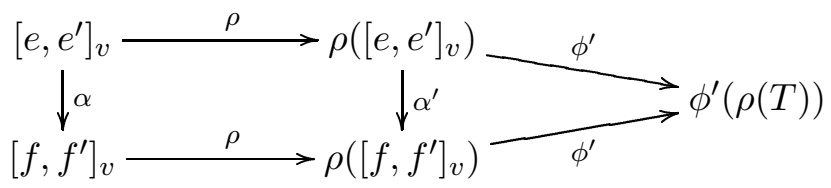

Now restrict $\alpha^{\prime}$ to the subtree $\left[\rho(e), \rho\left(e^{\prime}\right)\right]_{\rho(v)} \subseteq \rho\left(\left[e, e^{\prime}\right]_{v}\right)$ to obtain a graph isomorphism $\left[\rho(e), \rho\left(e^{\prime}\right)\right]_{\rho(v)} \rightarrow\left[\rho(f), \rho\left(f^{\prime}\right)\right]_{\rho(v)}$ which realizes the equivalence $\left(\rho(e), \rho\left(e^{\prime}\right)\right) \sim_{\phi^{\prime}}\left(\rho(f), \rho\left(f^{\prime}\right)\right)$. Thus, $\rho$ preserves the equivalence relation.

It follows that $\rho(S)$ supports all $\left(\rho(T), \rho(v), \phi^{\prime}\right)$-types. Note also that $\rho(S)$ has fewer edges than $S$. By repeating this factorization process at most $|E(S)|$ times, we obtain a finite composition of multi-folds $\rho$ and a morphism $\phi^{\prime}$ satisfying $\phi=\phi^{\prime} \rho$, such that $\left.\phi^{\prime}\right|_{\rho(S)}$ is an embedding and $\rho(S)$ supports all $\left(\rho(T), \rho(v), \phi^{\prime}\right)$-types. Then $\left.\phi^{\prime}\right|_{\rho(T)}$ is an embedding.

Proposition 4.11 Let $\psi: Z \rightarrow Y$ be an equivariant morphism of $G$-trees which induces an isomorphism of quotient graphs, and which preserves hyperbolicity of elements of $G$.

(a) If $e$ is any edge of $Y$ with $G_{\partial_{0} e} \supsetneq G_{e} \subsetneq G_{\partial_{1} e}$ then $\psi^{-1}(e)$ consists of a single edge.

(b) Assume further that $Y$ is reduced and $G \backslash Y$ has finitely many vertices. Then $\psi$ is a finite composition of parabolic folds.

Note that if every edge of $Y$ satisfies the condition in (a) then 4.11(a) implies that $\psi$ is an embedding. Then $\psi$ will be an isomorphism by equivariance and the assumption on quotient graphs.

Proof of 4.11(a) Suppose $Z$ contains edges $f$ and $f^{\prime}$ with $\psi(f)=\psi\left(f^{\prime}\right)=e$. First we reduce to the case where $\partial_{0} f=\partial_{0} f^{\prime}$. Apply the Multi-fold Lemma (4.8) to the subtree $\left[f, f^{\prime}\right]$ to obtain a factorization of $\psi$ :

$$
Z \stackrel{\rho_{1}}{\longrightarrow} Z_{1} \stackrel{\rho_{2}}{\longrightarrow} \cdots \stackrel{\rho_{k-1}}{\longrightarrow} Z_{k-1} \stackrel{\rho_{k}}{\longrightarrow} Z_{k} \longrightarrow Y
$$

where each $\rho_{i}$ is a multi-fold and $\rho_{k} \cdots \rho_{1}(f)=\rho_{k} \cdots \rho_{1}\left(f^{\prime}\right)$. Each map in this factorization induces an isomorphism of quotient graphs, because their composition does. Similarly, each map preserves hyperbolicity of elements of $G$, because equivariant maps cannot make elliptic elements hyperbolic. Take $i$ smallest so that $\rho_{i} \cdots \rho_{1}(f)=\rho_{i} \cdots \rho_{1}\left(f^{\prime}\right)$. Then $\partial_{0}\left(\rho_{i-1} \cdots \rho_{1}(f)\right)=\partial_{0}\left(\rho_{i-1} \cdots \rho_{1}\left(f^{\prime}\right)\right)$ or $\partial_{0}\left(\rho_{i-1} \cdots \rho_{1}(\bar{f})\right)=\partial_{0}\left(\rho_{i-1} \cdots \rho_{1}\left(\bar{f}^{\prime}\right)\right)$. We can now proceed with the argument using this pair of edges and the morphism $Z_{i-1} \rightarrow Y$. We have already remarked that the hypotheses of the proposition hold for the this map. 
Thus, without loss of generality, $\partial_{0} f=\partial_{0} f^{\prime}$. Since $\psi(f)=\psi\left(f^{\prime}\right)$ and $\psi$ induces an isomorphism on quotient graphs, $f$ and $f^{\prime}$ are in the same orbit, so there exists $\gamma \in G$ with $\gamma f=f^{\prime}$. Note that $\gamma \in G_{\partial_{0} f}-G_{f}$, and also $\gamma \in G_{e}$. Now choose an element $\sigma \in G_{\partial_{1} e}-G_{e}$. Suppose that $\sigma$ is in $G_{\partial_{1} f}-G_{f}$ as well. Then using the Hyperbolic Segment Condition (2.8) one finds that $\sigma \gamma$ is hyperbolic in $Z$. However, $\sigma$ and $\gamma$ are both in $G_{\partial_{1} e}$ so their product is elliptic in $Y$, giving the desired contradiction. Thus our strategy will be to pin down the behavior of $\sigma$ acting on $Z$.

Choose an element $\delta \in G_{\partial_{0} e}-G_{e}$. The product $\sigma \delta$ is hyperbolic in $Y$ by the Hyperbolic Segment Condition (2.8) and hence is hyperbolic in $Z$ by equivariance. Consider its axis $Z_{\sigma \delta} \subseteq Z$. The image $\psi\left(Z_{\sigma \delta}\right)$ is a $\langle\sigma \delta\rangle$-invariant subtree of $Y$ and therefore it contains the axis $Y_{\sigma \delta}$. Notice that $Y_{\sigma \delta}$ is the support of the following oriented path:

$$
\cdots \quad \delta^{-1} \bar{e}, \quad e, \sigma \bar{e}, \quad \sigma \delta e \cdots .
$$

Since $e \in Y_{\sigma \delta}$, the axis $Z_{\sigma \delta}$ contains an edge which is mapped by $\psi$ to $e$, and this edge must be in the same orbit as $f$; call it $\tau f$ where $\tau \in G$. Now, using equivariance, one sees that the set $Z_{\sigma \delta} \cap \psi^{-1}\left(Y_{\sigma \delta}\right)$ contains the following oriented edges, arranged coherently and in order along $Z_{\sigma \delta}$ (possibly with gaps in between):

$$
\cdots \quad \delta^{-1} \tau \bar{f}, \quad \tau f, \quad \sigma \tau \bar{f}, \quad \sigma \delta \tau f \quad \cdots .
$$

This shows that the edges $\tau f$ and $\sigma \tau f$ are incoherently oriented, and so $\sigma$ fixes a unique vertex $v \in[\tau f, \sigma \tau f] \subseteq Z_{\sigma \delta}$. Configuration (4.12) also shows that $[\tau f, \sigma \tau f]=\left[\partial_{0}(\tau f), \partial_{0}(\sigma \tau f)\right]$. Hence $\left[\partial_{0}(\tau f), v\right]=[\tau f, v]$, and $\sigma$ fixes no edge of this segment.

Having established the behavior of $\sigma$ we return our attention to $\gamma$, or rather to its conjugate $\tau \gamma \tau^{-1}$. This element fixes $\partial_{0}(\tau f)$ and takes $\tau f$ to $\tau f^{\prime}(\neq$ $\tau f$ ). Now $\sigma$ and $\tau \gamma \tau^{-1}$ satisfy the Hyperbolic Segment Condition (2.8) using the segment $[\tau f, v]$. This implies that $\sigma \tau \gamma \tau^{-1}$ is hyperbolic in $Z$. On the other hand, as $\psi(\tau f)=\psi(f)=e$, we must have $\tau \in G_{e}$ by equivariance. Then $\sigma, \tau$, and $\gamma$ are in the subgroup $G_{\partial_{1} e}$ and so $\sigma \tau \gamma \tau^{-1}$ is elliptic in $Y$, a contradiction.

Proof of 4.11(b) Suppose first that $G \backslash Y$ is a single loop. If the edges of $Y$ satisfy $G_{e} \neq G_{\partial_{0} e}$ then by $4.11(\mathrm{a})$ the map $\psi$ is an isomorphism. Otherwise suppose that $G_{f}=G_{\partial_{0} f}$ for some $f \in E(Y)$. Then $Y$ is parabolic and hence the elliptic elements form a subgroup of $G$ (see Remark 2.5). As $G \backslash Z$ is also a single loop, this implies that $Z$ is also parabolic (using the Hyperbolic Segment 
Condition (2.8)). Let $e \in E(Z)$ be an edge with image $f$ or $\bar{f}$, such that $G_{e}=G_{\partial_{0} e}$ and $\partial_{1} e=t \partial_{0} e$ for some $t \in G$ (for every edge $e \in E(Z)$, either $e$ or $\bar{e}$ has these last two properties, because $Z$ is parabolic).

We wish to know that $G_{\psi(e)}=G_{\partial_{0} \psi(e)}$. If $\psi(e)=f$ then this is already true. So suppose that $\psi(e)=\bar{f}$. By equivariance, we have that $\partial_{1} \bar{f}=t \partial_{0} \bar{f}$, ie, $\partial_{0} f=$ $t \partial_{1} f$. Hence $G_{t f} \subseteq G_{\partial_{1} t f}=G_{\partial_{0} f}=G_{f}$, which implies that $G_{t^{i+1} f} \subseteq G_{t^{i} f}$ for all $i$. Similarly, $G_{t^{i} e} \subseteq G_{t^{i+1} e}$ for all $i$, because $G_{e} \subseteq G_{\partial_{1} e}=G_{t \partial_{0} e}=G_{t e}$. These inclusions fit into a diagram as follows.

$$
\begin{aligned}
\cdots & \subseteq G_{t^{-1} e} \subseteq G_{e} \subseteq G_{t e} \subseteq \cdots \subseteq \bigcup_{n} G_{t^{n} e} \\
\bigcup_{n} G_{t^{n} f} \supseteq \cdots & \supseteq G_{t^{-1} f} \supseteq G_{f} \supseteq G_{t f} \supseteq \cdots \supseteq \bigcap_{n} G_{t^{n} f}
\end{aligned}
$$

The central vertical inclusions follow from equivariance of $\psi$, since $\psi\left(t^{i} e\right)=t^{i} \bar{f}$ and $G_{t^{i} \bar{f}}=G_{t^{i} f}$. The rightmost vertical inclusion is a consequence of all of the other inclusions in the diagram. Now observe that $\bigcup_{n} G_{t^{n} f}$ and $\bigcup_{n} G_{t^{n} e}$ are both equal to $\mathscr{E}$, the set of elliptic elements. In each case this follows from the facts that $t$ is hyperbolic (by Lemma 2.7) and the tree is parabolic. Therefore all of the inclusions in the bottom row of the diagram are equalities. This implies that $Y$ is a linear tree with all stabilizers equal to $\mathscr{E}$, as in Example 3.10.A. In particular, $G_{f}=G_{\partial_{1} f}$ as desired.

Renaming $\bar{f}$ as $f$ if necessary, we have: $\psi(e)=f, G_{e}=G_{\partial_{0} e}, G_{f}=G_{\partial_{0} f}$, $\partial_{1} e=t \partial_{0} e$, and $\partial_{1} f=t \partial_{0} f$. Now consider the subgroup $H=G_{f}$, and let $\sim$ be the relation on $X$ defined by (3.11) using $H, e$, and $t$. We claim that

$$
e_{0} \sim e_{1} \quad \text { if and only if } \psi\left(e_{0}\right)=\psi\left(e_{1}\right) .
$$

This equivalence implies that $\psi$ is a parabolic fold from $Z$ to its image. Since $Y$ is minimal (because it is reduced), this image is all of $Y$.

Note that both relations in (4.13) are equivariant, so in its proof we can take one of the edges to be $e$. So suppose that $\psi\left(e^{\prime}\right)=\psi(e)(=f)$. Then $e^{\prime}=\gamma e$ for some $\gamma \in G$, and $\gamma \in H$ by equivariance. Therefore $e \sim e^{\prime}$.

Next suppose that $e \approx e^{\prime}$, ie, that $e^{\prime}=h^{t^{-i}} e$ with $i \geqslant 0$ and $h \in H$. Then $\psi\left(e^{\prime}\right)=h^{t^{-i}} \psi(e)=h^{t^{-i}} f$. The properties $G_{f}=G_{\partial_{0} f}$ and $\partial_{1} f=t \partial_{0} f$ imply that $G_{t^{j} f} \subseteq G_{t^{k} f}$ whenever $j \leqslant k$. Thus $\left(G_{f}\right)^{t^{-i}}=G_{t^{-i} f} \subseteq G_{f}$, and so $h^{t^{-i}} \in G_{f}$. This implies that $\psi\left(e^{\prime}\right)=f=\psi(e)$, and so we have shown that $e_{0} \approx e_{1}$ implies $\psi\left(e_{0}\right)=\psi\left(e_{1}\right)$. Since the latter relation is transitive and $\sim$ is the transitive closure of $\approx$, it now follows that (4.13) holds. Therefore 4.11(b) is proved in the case where the quotient graph is a single loop. 
Next consider the general case. An edge $e \in E(Z)$ is called $\psi$-isolated if no two edges of $\psi^{-1} \psi(e)$ have a common endpoint. As $Y$ is reduced, 4.11(a) implies that every edge that is not $\psi$-isolated projects to a loop in $G \backslash Z$. We are interested in $\psi$-isolated edges because of the following observation: if $\psi: Z \rightarrow$ $Y$ is any morphism of trees such that every edge of $Z$ is $\psi$-isolated, then $\psi$ is an embedding. This is true because otherwise, $\psi$ would factor through a nontrivial fold, and then the edge where the fold is performed would not be $\psi$-isolated.

Suppose that $e \in E(Z)$ is an edge which is not $\psi$-isolated. Let $f=\psi(e)$, and let $e$ be oriented so that $G_{e}=G_{\partial_{0} e}$. Let $T \subseteq Z$ be the connected component of $G e$ containing $e$. Then $T$ is a parabolic $G_{T}$-tree with quotient graph a single loop ( $G_{T}$ is the stabilizer of $T$ ). Let $S \subseteq Y$ be the connected component of $G f$ containing $f$. Then $S$ is also a $G_{T}$-tree, and $\left.\psi\right|_{T}: T \rightarrow S$ is a $G_{T}$-equivariant map inducing an isomorphism of quotient graphs. By the previous analysis, $\left.\psi\right|_{T}$ is a parabolic fold of $G_{T}$-trees. In fact it is the parabolic fold performed at $e$ using the subgroup $H=\left(G_{T}\right)_{f}$.

Let $\sim_{T}$ denote the relation on $T$ given by this parabolic fold, and let $\sim$ be its equivariant extension to $Z$. Then $\sim$ is simply the parabolic fold in $Z$ performed at $e$ using $H$. We have that $e_{0} \sim_{T} e_{1}$ if and only if $\psi\left(e_{0}\right)=\psi\left(e_{1}\right)$, for edges $e_{0}, e_{1} \in E(T)$. Since $\psi$ is equivariant, we also have that $e_{0} \sim e_{1}$ implies $\psi\left(e_{0}\right)=\psi\left(e_{1}\right)$ for all $e_{0}, e_{1} \in E(Z)$. It follows that $\psi$ factors as $Z \stackrel{\rho}{\rightarrow} Z^{\prime} \stackrel{\psi^{\prime}}{\rightarrow} Y$, where $\rho$ is the parabolic fold defined by $\sim$. Note that $\left.\psi^{\prime}\right|_{\rho(T)}: \rho(T) \rightarrow S$ is an isomorphism. In particular the parabolic fold $\rho$ is nontrivial, since $\left.\psi\right|_{T}: T \rightarrow S$ is not an isomorphism (as $e$ is not $\psi$-isolated).

Recall that in a parabolic fold at $e$, all identifications of vertices occur inside connected components of $G e$. Therefore edges and vertices in different components remain in different components. Now let $e^{\prime}=\rho(e) \in E\left(Z^{\prime}\right)$. We do not know whether $\psi^{\prime-1} \psi^{\prime}\left(e^{\prime}\right)=\left\{e^{\prime}\right\}$, but no two edges of $\psi^{\prime-1} \psi^{\prime}\left(e^{\prime}\right)$ are in the same connected component of $G e^{\prime}$, and therefore $e^{\prime}$ is $\psi^{\prime}$-isolated.

Thus, by factoring through a parabolic fold, any edge may be made $\psi^{\prime}$-isolated. During this parabolic fold, the other edges are affected as follows. All translates of $\rho(e)$ and $\rho(\bar{e})$ are $\psi^{\prime}$-isolated. If $f$ is a $\psi$-isolated edge of $Z$ such that $\partial_{0} f, \partial_{1} f \notin G \partial_{0} e$, then $\rho(f)$ is $\psi^{\prime}$-isolated, as $\rho$ does not affect $Z$ away from $G e$. If $f$ is a $\psi$-isolated edge of $Z$ with $\partial_{0} f, \partial_{1} f \in G \partial_{0} e$ and $f \notin(G e \cup G \bar{e})$, then $G_{\partial_{0} \rho(f)} \supsetneq G_{\rho(f)} \subsetneq G_{\partial_{1} \rho(f)}$. This is true because $G_{\partial_{0} \rho(e)}$ is strictly larger than $G_{\partial_{0} e}$, as $\rho$ is nontrivial, and $G_{\rho(f)}=G_{f}$ (because $f \notin(G e \cup G \bar{e})$ ). Thus, $\rho(f)$ is $\psi^{\prime}$-isolated by 4.11(a). Lastly, if only one of $\partial_{0} f, \partial_{1} f$ is in the orbit 
$G \partial_{0} e$, then $\rho(f)$ does not project to a loop in $G \backslash Z^{\prime}$, and hence is $\psi^{\prime}$-isolated by $4.11(\mathrm{a})$.

These remarks imply that $\psi$ factors through a composition of finitely many nontrivial parabolic folds, at most one for each vertex orbit, after which all edges are $\psi^{\prime}$-isolated. Here the factorization is $Z \stackrel{\rho}{\rightarrow} Z^{\prime} \stackrel{\psi^{\prime}}{\rightarrow} Y$, where $\rho$ now represents the sequence of parabolic folds. Then $\psi^{\prime}$ is an embedding, and it is an isomorphism because $Y$ is minimal.

Notice that the parabolic folds in 4.11(b) do not interfere with each other and may be performed in any order. Thus a similar result holds in the case where $G \backslash Y$ has infinitely many vertices, if one is willing to perform infinitely many "disjoint" parabolic folds simultaneously.

Next we give a proof of Proposition 3.15, using the Multi-fold Lemma. Recall that this proposition characterizes parabolic folds which admit finite factorizations into multi-folds.

Proof of Proposition 3.15 We are given a parabolic fold $\rho: X \rightarrow Y$, performed at $e \in E(X)$. Suppose that $G_{\rho(e)}$ fixes a vertex $v \in V(X)$. Let $T \subseteq X$ be the subtree spanned by the edges $\rho^{-1} \rho(e)$. We claim that $T$ has finite diameter. If $e^{\prime} \in \rho^{-1} \rho(e)$ then $e^{\prime}=\gamma e$ for some $\gamma \in G$ (because parabolic folds induce isomorphisms on quotient graphs). Then $\gamma \rho(e)=\rho(\gamma e)=\rho\left(e^{\prime}\right)=\rho(e)$, so $\gamma \in G_{\rho(e)} \subseteq G_{v}$. We now have $\gamma([v, e])=\left[v, e^{\prime}\right]$ and hence $d\left(e, e^{\prime}\right) \leqslant 2 d(e, v)$ (as $[e, v] \cup\left[v, e^{\prime}\right]$ is a path joining $e$ to $e^{\prime}$ ). This shows that $\rho^{-1} \rho(e)$ has diameter at most $4 d(e, v)$, and the same must hold for $T$.

Now, applying the Multi-fold Lemma (4.8) using the subtree $T$, we obtain a factorization of $\rho$ as $X \stackrel{\rho^{\prime}}{\rightarrow} X^{\prime} \stackrel{i}{\rightarrow} Y$. Here $\rho^{\prime}$ is a finite composition of multifolds and $\left.i\right|_{\rho^{\prime}(T)}$ is an embedding. Thus, $i^{-1}(\rho(e))$ is a single edge. The same is true of translates of $e$ by equivariance, and also of other geometric edges, since the original parabolic fold $\rho$ is bijective away from the orbit of $e$. This shows that $i$ is an isomorphism, and hence $\rho$ has been factored into a finite composition of multi-folds.

To see that the multi-folds are of type II, note that their composition induces an isomorphism on quotient graphs, and hence each multi-fold does as well.

Finally consider the converse: suppose that the parabolic fold $\rho$ is a finite composition of multi-folds (which must be of type II). Note that these multifolds occur along edges $f \in G \bar{e}$, so that $G_{f}=G_{\partial_{1} f}$. It follows that these multi-folds preserve hyperbolicity of elements of $G$, so by Proposition 3.16, $\rho$ 
is an elementary deformation. Now, by Remark 3.5, every stabilizer in $Y$ fixes a vertex of $X$. In particular, $G_{\rho(e)}$ fixes some $v \in V(X)$.

Lemma 4.14 Let $X$ be a reduced $G$-tree. If $x$ and $y$ are distinct vertices of $X$ with $G_{x} \subseteq G_{y}$ then there is a hyperbolic element $t \in G$ such that $G_{x} \subseteq\left(G_{x}\right)^{t}$.

Proof We are given that $G_{x}$ fixes $y$. Thus $G_{x}$ fixes the path from $x$ to $y$, including the first edge $e$ on this path, and so $G_{x}=G_{e}\left(\right.$ where $\left.\partial_{0} e=x\right)$. Since $X$ is reduced, there is an element $t$ of $G$ taking $\partial_{0} e$ to $\partial_{1} e$. Then $G_{x}=G_{e} \subseteq G_{\partial_{1} e}=\left(G_{x}\right)^{t}$. The element $t$ is hyperbolic by Lemma 2.7 , using either of the first two criteria.

Lemma 4.15 Let $Y$ be a $G$-tree and suppose $s, t \in G$ are elements with $t$ hyperbolic. Let $x$ and $y$ be any two vertices of $Y$. Then $d\left(x, s t^{n} y\right) \rightarrow \infty$ as $n \rightarrow \infty$.

Proof Note that $\ell_{Y}(t)>0$ and that $t^{-n}$ has translation length $n \cdot \ell_{Y}(t)$ for positive $n$. Thus:

$$
\begin{aligned}
d\left(x, s t^{n} y\right)=d\left(t^{-n} s^{-1} x, y\right) & \geqslant d\left(t^{-n} s^{-1} x, s^{-1} x\right)-d\left(s^{-1} x, y\right) \\
& \geqslant n \cdot \ell_{Y}(t)-d\left(s^{-1} x, y\right) .
\end{aligned}
$$

Proposition 4.16 Let $X$ and $Y$ be cocompact $G$-trees which define the same partition of $G$ into elliptic and hyperbolic elements. Suppose further that $X$ is reduced and every vertex stabilizer of $X$ fixes a vertex of $Y$. Then, after possibly subdividing $X$, there is an equivariant morphism $\phi: X \rightarrow Y$.

Proof We will define the morphism $\phi$ in the following manner. First we define $\phi$ on a finite set of vertices of $X$, one representing each vertex orbit. Having done this correctly, the map then extends equivariantly to $V(X)$ in a unique way. Once the map is defined on $V(X)$, each edge $e$ is sent to the unique path in $Y$ joining $\phi\left(\partial_{0} e\right)$ to $\phi\left(\partial_{1} e\right)$. We need to be certain that these two vertices are not the same. Then $e$ is subdivided so that preimages of vertices in $Y$ are vertices in $X$, and we have an equivariant morphism $\phi: X^{\prime} \rightarrow Y$ (here $X^{\prime}$ is $X$, after subdivision).

Let $S \subseteq X$ be a subtree whose edges map bijectively to the edges of $G \backslash X$. For each vertex $v$ of $S$, the stabilizer $G_{v}$ may or may not be maximal among the elliptic subgroups of $G$. Label the vertices of $S$ as $v_{1}, \ldots, v_{k}$ so that the vertices having maximal elliptic stabilizers occur first in the list. Also arrange that all 
vertices in the same orbit occur consecutively. Consider the $i^{\text {th }}$ orbit arising in the list. It has a first vertex $v_{i_{0}}$ and various translates $v_{i_{0}+q}$ for $1 \leqslant q \leqslant r_{i}$. Set $\gamma_{i_{0}}=1$ and choose elements $\gamma_{i_{0}+q} \in G$ such that $\gamma_{i_{0}+q} v_{i_{0}}=v_{i_{0}+q}$ for each $q$.

Thus, whenever $v_{i}$ and $v_{j}$ are in the same orbit, there is a chosen element $\gamma_{i} \gamma_{j}^{-1}$ taking $v_{j}$ to $v_{i}$. Also, for each $j$ the element $\gamma_{j}^{-1}$ takes $v_{j}$ to a chosen representative of its orbit. If $i \neq j$ then $\gamma_{i} \gamma_{j}^{-1}$ is hyperbolic, by Lemma 2.7(b).

We wish to define $\phi$ on $\left\{v_{1}, \ldots, v_{k}\right\}$ with the following properties:

(i) $\phi$ is injective,

(ii) $G_{v_{i}} \subseteq G_{\phi\left(v_{i}\right)}$ for each $i$,

(iii) whenever $v_{i}$ and $v_{j}$ are in the same orbit, $\gamma_{i} \gamma_{j}^{-1} \phi\left(v_{j}\right)=\phi\left(v_{i}\right)$.

We proceed one orbit at a time. Suppose that $\phi$ has already been defined on $\left\{v_{1}, \ldots, v_{d-1}\right\}$, satisfying all three properties. Consider $v_{d}$ and its translates $v_{d+1}, \ldots, v_{d+r}$. The stabilizer $G_{v_{d}}$ fixes a vertex $w$ of $Y$. If

$$
\left\{\phi\left(v_{1}\right), \ldots, \phi\left(v_{d-1}\right)\right\} \cap\left\{\gamma_{d} w, \ldots, \gamma_{d+r} w\right\}=\varnothing
$$

then we can define $\phi\left(v_{d+q}\right)=\gamma_{d+q} w$ for each $q \leqslant r$. Properties (i)-(iii) are satisfied in this case. However, (4.17) might not hold. So suppose that $\phi\left(v_{p}\right)=$ $\gamma_{d+q} w$ for some $p \leqslant d-1$ and $0 \leqslant q \leqslant r$.

We consider two cases: either $G_{v_{p}}$ is maximal among elliptic subgroups, or $G_{v_{d+q}}$ is not maximal elliptic. The third possibility, that $G_{v_{d+q}}$ is maximal elliptic and $G_{v_{p}}$ is not, was ruled out when we ordered the vertices. In the first case note that $G_{v_{p}} \subseteq G_{\gamma_{d+q} w}$ by property (ii) for $v_{p}$, and by maximality of $G_{v_{p}}$ these stabilizers are equal. Also, since $G_{v_{d}} \subseteq G_{w}$, conjugation by $\gamma_{d+q}$ yields $G_{v_{d+q}} \subseteq G_{\gamma_{d+q} w}$. Therefore $G_{v_{d+q}} \subseteq G_{v_{p}}$. In the second case, as $G_{v_{d+q}}$ is not maximal elliptic, there is an elliptic subgroup $H$ properly containing $G_{v_{d+q}}$. Since $H$ is elliptic it is contained in $G_{v}$ for some $v$ and we have $G_{v_{d+q}} \subsetneq H \subseteq$ $G_{v}$.

In each of the two cases we have found that $G_{v_{d+q}}$ is contained in the stabilizer of a vertex of $X$ other than $v_{d+q}$. By Lemma 4.14, there must be a hyperbolic element $t^{\prime} \in G$ such that $G_{v_{d+q}} \subseteq\left(G_{v_{d+q}}\right)^{t^{\prime}}$. Now observe that

$$
\begin{aligned}
G_{v_{d}}=\left(G_{v_{d+q}}\right)^{\gamma_{d+q}-1} & \subseteq\left(\left(G_{v_{d+q}}\right)^{t^{\prime}}\right)^{\gamma_{d+q}}{ }^{-1} \\
& =\left(\left(\left(G_{v_{d}}\right)^{\gamma_{d+q}}\right)^{t^{\prime}}\right)^{\gamma_{d+q}-1}=\left(G_{v_{d}}\right)^{\gamma_{d+q}{ }^{-1} t^{\prime} \gamma_{d+q}} .
\end{aligned}
$$

We can simplify forthcoming notation by setting $t=\gamma_{d+q}{ }^{-1} t^{\prime} \gamma_{d+q}$, so that we have $G_{v_{d}} \subseteq\left(G_{v_{d}}\right)^{t}$. This inclusion will allow us to replace $w$ by some $t^{n} w$ to arrange that (4.17) holds. Note that $t$ is hyperbolic. 
For any pair of indices $p \leqslant d-1$ and $q \leqslant r$ consider the vertices $\phi\left(v_{p}\right)$ and $w$, and the element $s=\gamma_{d+q}$. Applying Lemma 4.15 with this data we find that

$$
d\left(\phi\left(v_{p}\right), \gamma_{d+q} t^{n} w\right)>0
$$

for sufficiently large $n$. Fix $n$ so that (4.18) holds for every $p \leqslant d-1$ and $q \leqslant r$. Now define $\phi\left(v_{d+q}\right)=\gamma_{d+q} t^{n} w$ for each $q \leqslant r$.

Trivially, property (iii) holds. For property (i) note first that since each $\gamma_{i} \gamma_{j}^{-1}$ is hyperbolic, $\phi$ is injective on the set $\left\{v_{d}, \ldots, v_{d+r}\right\}$. It is injective on the set $\left\{v_{1}, \ldots, v_{d-1}\right\}$ by assumption, and inequality (4.18) implies that the $\phi$-images of these two sets are disjoint. Thus $\phi$ is injective. Property (ii) holds as follows. For each $q \leqslant r$ we have

$$
\begin{aligned}
G_{v_{d+q}} & =\left(G_{v_{d}}\right)^{\gamma_{d+q}} \\
& \subseteq\left(G_{v_{d}}\right)^{\gamma_{d+q} t^{n}} \\
& \subseteq\left(G_{w}\right)^{\gamma_{d+q} t^{n}} \\
& =G_{\gamma_{d+q} t^{n} w}=G_{\phi\left(v_{d+q}\right)} .
\end{aligned}
$$$$
\text { as } G_{v_{d}} \subseteq\left(G_{v_{d}}\right)^{t}
$$$$
\text { as } G_{v_{d}} \text { fixes } w
$$

Thus $\phi$ satisfies (i)-(iii), and proceeding inductively on orbits, such a $\phi$ can be defined on all of $\left\{v_{1}, \ldots, v_{k}\right\}$.

Properties (ii) and (iii) imply that $\phi$ has a (unique) equivariant extension to $V(X)$. Now consider any edge $e \in E(S)$, with endpoints $v_{i_{0}}, v_{i_{1}}$. Property (i) implies that $\phi\left(v_{i_{0}}\right) \neq \phi\left(v_{i_{1}}\right)$ and so $e$ can be mapped to a nontrivial embedded path in $Y$. By equivariance this holds for every edge of $X$, as $S$ meets every edge orbit. Therefore the equivariant morphism $\phi: X^{\prime} \rightarrow Y$ can be constructed as required.

Proof of Theorem 4.2 We have seen in Remark 3.5 that elementary moves do not change the set of elliptic subgroups of $G$. Thus one implication is clear. For the other implication, we are given cocompact $G$-trees $X$ and $Y$ with the same elliptic subgroups. In particular $X$ and $Y$ define the same partition of $G$ into elliptic and hyperbolic elements. We can assume without loss of generality that $X$ and $Y$ are reduced (by performing collapse moves on both).

By Proposition 4.16, there is an equivariant morphism $\phi: X^{\prime} \rightarrow Y$, where $X^{\prime}$ is the result of subdividing $X$. Note that since subdivision is a special case of an expansion move, it suffices for us to show that $X^{\prime}$ and $Y$ are related by a deformation. As $Y$ is reduced, it is minimal, and hence $\phi$ is surjective. By Proposition 4.7, $\phi$ factors as $\psi \rho$, where $\psi$ induces an isomorphism on quotient graphs and $\rho$ is a finite composition of folds. By Proposition 4.11(b) the map 
$\psi$ factors as a finite composition of parabolic folds, as $\psi=\rho_{k} \cdots \rho_{0}$. Now the original map $\phi$ has the form

$$
X^{\prime} \stackrel{\rho}{\longrightarrow} Z_{0} \stackrel{\rho_{0}}{\longrightarrow} Z_{1} \stackrel{\rho_{1}}{\longrightarrow} \cdots \stackrel{\rho_{k-1}}{\longrightarrow} Z_{k} \stackrel{\rho_{k}}{\longrightarrow} Y .
$$

Suppose the parabolic fold $\rho_{k}$ is performed at $e \in E\left(Z_{k}\right)$. Consider the stabilizer $G_{\rho_{k} e}$ in $Y$. By our assumptions on $X$ and $Y$, this subgroup has a fixed point $v \in V\left(X^{\prime}\right)$. By equivariance of the maps, $G_{v}$ fixes the vertex $\rho_{k-1} \cdots \rho_{0} \rho v$ of $Z_{k}$, and hence $G_{\rho_{k} e}$ fixes a vertex of $Z_{k}$. Now we can apply Proposition 3.15 to the map $\rho_{k}$ and factor it into a finite composition of type II multi-folds. Repeating this argument for each $\rho_{i}$, we conclude that $\phi$ factors into a finite composition of folds and type II multi-folds.

Recall that the map $\phi$ preserves hyperbolicity of elements of $G$. It follows that each individual fold or multi-fold also has this property, as equivariant maps cannot make elliptic elements hyperbolic. Therefore, applying Proposition 3.16 to each fold and multi-fold, $\phi$ is a finite composition of elementary moves. This concludes the proof of the theorem.

\section{Local Rigidity}

Definition 5.1 A $G$-tree is proper if $G_{e} \subsetneq G_{\partial_{0} e}$ for every edge $e$. This is equivalent to the property " $V(X)$ is a strict $G$-set" from [4]. Notice that proper $G$-trees are reduced and minimal.

A $G$-tree is slide-free if it is minimal and, for all edges $e$ and $f$ with $\partial_{0} e=\partial_{0} f$, $G_{e} \subseteq G_{f}$ implies $f \in G e$ or $f \in G \bar{e}$. A quick look at Definition 3.6 will confirm that a minimal $G$-tree is slide-free if and only if it admits no slide moves.

A $G$-tree is strongly slide-free if it is minimal and, for all edges $e$ and $f$ with $\partial_{0} e=\partial_{0} f, G_{e} \subseteq G_{f}$ implies $f \in G e$. Consider for example the graph of groups with underlying graph a loop, with edge and vertex groups $\mathbb{Z}$, and with inclusion maps multiplication by 2 and 4 . The corresponding tree is slide-free because there is only one geometric edge orbit. However, it is not strongly slide-free.

Remark 5.2 Strongly slide-free trees are proper, as follows. Let $X$ be a strongly slide-free $G$-tree and let $e \in E(X)$ be any edge. Minimal trees have no terminal edges, so there is an edge $e^{\prime} \neq e$ with $\partial_{0} e^{\prime}=\partial_{0} e$. If $G_{e}=G_{\partial_{0} e}$ then $G_{e^{\prime}} \subseteq G_{e}$, and so $e^{\prime}=\gamma e$ for some $\gamma \in G$. But then $\gamma \in G_{\partial_{0} e}-G_{e}$, a contradiction. Therefore $G_{e} \subsetneq G_{\partial_{0} e}$. 
Throughout this section, $X$ is a $G$-tree which possesses a maximal stabilizer. Every proper $G$-tree has this property, for example. The set of maximal stabilizers is called $M(X)$. If $V \in M(X)$ then $X_{V}$ is the tree of fixed points of $V$, called the characteristic subtree of $V$. Note that each $X_{V}$ is nonempty and $X_{V} \cap X_{W}=\varnothing$ for all distinct pairs $V, W \in M(X)$. Let $[V, W]$ denote the unique smallest segment joining $X_{V}$ to $X_{W}$. Note that $[V, W]$ has positive length, and meets $X_{V}$ and $X_{W}$ only in its endpoints.

If $G$ contains a hyperbolic element then there is a unique minimal $G$-invariant subtree $X_{G}$, which is nonempty (see $[1,7.5]$ ). This occurs if $X$ has two or more maximal stabilizers, by the Hyperbolic Segment Condition (2.8). Otherwise, if $X$ has one maximal stabilizer and there are no hyperbolic elements, then $G$ has a global fixed point by Proposition 2.6. In this case we define $X_{G}$ to be the tree of fixed points of $G$, in keeping with the definition of $X_{V}$ above.

Lemma 5.3 If $e \in E(X)-E\left(X_{G}\right)$ separates $\partial_{0} e$ from $X_{G}$, then $G_{e}=G_{\partial_{0} e}$.

Proof Note that every $\gamma \in G$ maps $X_{G}$ to itself. Then if $\gamma$ fixes $\partial_{0} e$, it also fixes the path from $\partial_{0} e$ to $X_{G}$, which contains $e$. Thus $G_{\partial_{0} e} \subseteq G_{e}$.

Definition 5.4 A segment $\left(e_{1}, \ldots, e_{n}\right)$ is monotone if either $G_{e_{i}}=G_{\partial_{0} e_{i}}$ for all $i$, or $G_{\bar{e}_{i}}=G_{\partial_{0} \bar{e}_{i}}$ for all $i$. We say that $\partial_{0} e_{1}$ is minimal and $\partial_{1} e_{n}$ is maximal in the first case, and the reverse in the second case. An endpoint may be both minimal and maximal; this occurs if and only if all stabilizers along the segment are equal. We also declare every segment of length zero to be monotone.

A segment $[v, w]$ is injective if its interior maps injectively to $G \backslash X$. A segment $[v, w]$ is called a telescope if there is an edge $e \in[v, w]$ which separates $[v, w]$ into two monotone segments, in which $\partial_{0} e$ and $\partial_{1} e$ are minimal. Note that telescopes have positive length.

Such an edge $e$ is called a $(v, w)$-minimal edge. Note that $\bar{e}$ is also $(v, w)-$ minimal. If $[v, w]$ is a telescope then an edge $e \in[v, w]$ is $(v, w)$-minimal if and only if $G_{e}=G_{v} \cap G_{w}$. The set of $(v, w)$-minimal edges forms a nontrivial sub-segment of $[v, w]$, possibly equal to all of $[v, w]$. If $[v, w]=[V, W]$ with $V, W \in M(X)$, a $(v, w)$-minimal edge is also called a $(V, W)$-minimal edge.

A proper telescope is a telescope $[v, w]$ that is not monotone: $G_{v} \nsubseteq G_{w}$ and $G_{w} \not \subset G_{v}$.

In the next definition we consider a pair of $G$-trees. Suppose that $Z$ is a proper $G$-tree and $X$ is obtained from $Z$ by an elementary deformation. In 
the transition from $Z$ to $X$, various edges may have stretched into telescopes. Also, vertices may have expanded into subtrees whose vertices and edges have a common stabilizer (these are the characteristic subtrees $X_{U}$ ). Other changes are likely to have occurred as well. We would like to know that the pattern of these telescopes and characteristic subtrees has the same combinatorics as $Z$. For this reason we must eventually make the assumption that $Z$ is strongly slide-free.

The definition encodes what is meant by the pattern of telescopes having the same combinatorics as $Z$. The somewhat elaborate requirements, taken together, are invariant under elementary moves if $Z$ is strongly slide-free. This is the content of Proposition 5.15. The requirements also imply that $Z$ can be recovered simply by performing collapse moves. In this way Theorem 1.2 is proved.

Definition 5.5 Let $X$ be a $G$-tree that possesses a maximal stabilizer. Suppose that $Z$ is a $G$-tree with vertex set $M(X)$, with the same $G$-action (by conjugation). Note that $Z$ must be proper. We say that $X$ is a proper telescoping of $Z$ if:

(i) for every pair $V, W \in M(X)$ that bounds an edge in $Z$, the segment $[V, W]$ is a telescope (call $[V, W]$ a $Z$-telescope);

(ii) each $Z$-telescope $[V, W]$ is the union of two injective telescopes $[V, w]$ and $[v, W]$, whose intersection $[v, w]$ is a proper telescope, such that either $[v, w]=[V, W]$ or $[w, W]=t([v, V])$ for some $t \in G$;

(iii) for every $Z$-telescope $[V, W]$, the sub-telescope $[v, w]$ given by (ii) contains a $(V, W)$-minimal edge $e_{V W}$ which is not contained in any other $Z$-telescope (call $e_{V W}$ a $Z$-minimal edge).

If we drop the requirement in (ii) that $[v, w]$ is proper, then $X$ is called a telescoping of $Z$.

The meaning of condition (ii) is that each $Z$-telescope has one of two configurations. Either it is proper and injective (the case $[v, w]=[V, W]$ ), or its image in the quotient graph is a "lasso," a path which travels along a segment, around a circuit, then back along the initial segment. Notice that the sub-telescope $[v, w] \subseteq[V, W]$ is uniquely determined, as the maximal injective telescope which is positioned symmetrically along $[V, W]$. It maps to the "circuit" part of the lasso. Note that the $Z$-minimal edge $e_{V W}$ in (iii) is simultaneously a minimal edge for the telescopes $[V, W],[V, w],[v, W]$, and $[v, w]$. 
Note that every proper $G$-tree is a proper telescoping of itself. Also, in any proper tree, telescopes have length 1 . Hence if $X$ is a telescoping of $Z$ and $X$ is proper then $X \cong Z$.

Lemma 5.6 If $X$ is a telescoping of $Z$ then

$$
X_{G}=\bigcup_{U \in M(X)}\left(X_{G} \cap X_{U}\right) \cup \bigcup\{Z \text {-telescopes }\} .
$$

Proof First note that

$$
X_{G} \subseteq \bigcup_{U \in M(X)} X_{U} \cup \bigcup\{Z \text {-telescopes }\}
$$

because the right hand side is a $G$-invariant subtree of $X$ (equal to the subtree spanned by the $X_{U}$ 's). To prove the lemma it remains to show that every $Z$ telescope is contained in $X_{G}$. Note that every $Z$-telescope has the property that its endpoint stabilizers are strictly larger than any of its edge stabilizers (recall that $[V, W]$ meets $X_{V}$ and $X_{W}$ only in its endpoints). The Hyperbolic Segment Condition (2.8) shows that such a segment is contained in the axis of a hyperbolic element. Since $X_{G}$ contains every hyperbolic axis, we are done.

Lemma 5.7 Let $X$ be a telescoping of $Z$, and let $[V, W]$ and $\left[V^{\prime}, W^{\prime}\right]$ be $Z$-telescopes. If $\{V, W\} \cap\left\{V^{\prime}, W^{\prime}\right\}=\varnothing$ then $[V, W] \cap\left[V^{\prime}, W^{\prime}\right]=\varnothing$.

This implies that if two $Z$-telescopes meet then they have a common endpoint. The lemma is a topological consequence of property 5.5 (iii).

Proof Suppose that $\{V, W\} \cap\left\{V^{\prime}, W^{\prime}\right\}=\varnothing$. The geometric edges $(V, W)$ and $\left(V^{\prime}, W^{\prime}\right)$ in $Z$ are separated by at least one edge. Consider the smallest segment in $Z$ containing these two edges. Without loss of generality assume that its vertices, in order, are $\left(W, V, U_{1}, \ldots, U_{k}, V^{\prime}, W^{\prime}\right)$ for some $k \geqslant 0$.

Let $T_{i} \subseteq X$ be the smallest tree containing

$$
\left(X_{W} \cup X_{V} \cup X_{V^{\prime}} \cup X_{W^{\prime}}\right) \cup\left(X_{U_{1}} \cup \cdots \cup X_{U_{i}}\right)
$$

for each $i \geqslant 1$, and let $T_{0}$ be the span of $\left(X_{W} \cup X_{V} \cup X_{V^{\prime}} \cup X_{W^{\prime}}\right)$. Then each $T_{i}$ is the union of the characteristic subtrees in (5.8) and the $Z$-telescopes joining them. More specifically, for each $i \geqslant 1$ we have that

$$
T_{i+1}=T_{i} \cup\left[U_{i}, U_{i+1}\right] \cup X_{U_{i+1}},
$$

as the right hand side is certainly a (connected) tree containing the required subtrees. Similarly, $T_{1}=T_{0} \cup\left[V, U_{1}\right] \cup X_{U_{1}}$. 
Now suppose that $[V, W] \cap\left[V^{\prime}, W^{\prime}\right] \neq \varnothing$. This implies that

$$
T_{0}=\left(X_{W} \cup X_{V} \cup X_{V^{\prime}} \cup X_{W^{\prime}}\right) \cup[W, V] \cup\left[V^{\prime}, W^{\prime}\right] .
$$

Together with the previous remarks we now have that

$$
\begin{aligned}
T_{k}= & \left(X_{W} \cup X_{V} \cup X_{V^{\prime}} \cup X_{W^{\prime}}\right) \cup\left(X_{U_{1}} \cup \cdots \cup X_{U_{k}}\right) \\
& \cup\left([W, V] \cup\left[V^{\prime}, W^{\prime}\right]\right) \cup\left(\left[V, U_{1}\right] \cup\left[U_{1}, U_{2}\right] \cup \cdots \cup\left[U_{k-1}, U_{k}\right]\right) .
\end{aligned}
$$

Consider the $Z$-telescope $\left[U_{k}, V^{\prime}\right]$. It is contained in $T_{k}$ and its interior meets no $X_{U}, U \in M(X)$. Therefore equation (5.9) implies that

$$
\left[U_{k}, V^{\prime}\right] \subseteq\left([W, V] \cup\left[V^{\prime}, W^{\prime}\right]\right) \cup\left(\left[V, U_{1}\right] \cup\left[U_{1}, U_{2}\right] \cup \cdots \cup\left[U_{k-1}, U_{k}\right]\right) .
$$

Thus every edge of $\left[U_{k}, V^{\prime}\right]$ is contained in another $Z$-telescope, contradicting property 5.5(iii).

Lemma 5.10 Let $X$ be a telescoping of $Z$, with $Z$ strongly slide-free. Then $X$ is a proper telescoping of $Z$.

Proof Let $[V, W]$ be a $Z$-telescope with injective sub-telescope $[v, w]$. If $[v, w]=[V, W]$ then it is proper because $V$ and $W$ are maximal. If $t([V, v])=$ $[W, w]$ for some $t \in G$ and $G_{v} \subseteq G_{w}$, then $G_{v}=V \cap W$ because $[v, w]$ contains a $(V, W)$-minimal edge. As $t(v)=w$, we also have $G_{w}=\left(V^{t} \cap W^{t}\right)$. Let $e \in E(Z)$ be the edge with initial vertex $V$ and terminal vertex $W$. Then te has initial vertex $W$, and hence $\bar{e}, t e \in E_{0}(W)$. Note that $G_{\bar{e}}=V \cap W=G_{v}$ and $G_{t e}=V^{t} \cap W^{t}=G_{w}$, so we have $G_{\bar{e}} \subseteq G_{t e}$. As $Z$ is strongly slide-free, $\bar{e} \in G t e=G e$. But then $e$ is inverted by an element of $G$, a contradiction. Thus $G_{v} \subseteq G_{w}$ cannot hold, and similarly $G_{w} \not \subset G_{v}$, so $[v, w]$ is proper.

Lemma 5.11 Let $X$ be a telescoping of $Z$, with $Z$ strongly slide-free. Suppose that $e$ is a $Z$-minimal edge with $G_{e}=G_{\partial_{0} e}$. Then $\partial_{0} e$ is contained in exactly one $Z$-telescope.

Proof Suppose that $e$ is $Z$-minimal for the $Z$-telescope $[V, W]$. If $\partial_{0} e$ is contained in another $Z$-telescope, then by Lemma 5.7 the two telescopes have a common endpoint. Suppose the second $Z$-telescope is $\left[V, W^{\prime}\right]$. Then since $G_{\partial_{0} e}=G_{e}=V \cap W$ and $\partial_{0} e \in\left[V, W^{\prime}\right]$, we must have $(V \cap W) \supseteq\left(V \cap W^{\prime}\right)$, as $\left[V, W^{\prime}\right]$ is a telescope.

Now since $Z$ is strongly slide-free, $V=V^{\delta}$ and $W^{\prime}=W^{\delta}$ for some $\delta \in G$. Then $\delta([V, W])=\left[V, W^{\prime}\right]$, fixing the endpoint in $X_{V}$. Since $\partial_{0} e \in[V, W] \cap\left[V, W^{\prime}\right]$, $\partial_{0} e$ is fixed by $\delta$. But $\delta e \neq e$ as $e \notin\left[V, W^{\prime}\right]$, and hence $\delta \in G_{\partial_{0} e}-G_{e}$, a contradiction. 
The rest of this section deals with invariance of properties under an elementary move from $X$ to $Y$. Without ambiguity, we will sometimes use the same symbol to denote an edge or vertex of $X$ and its image in $Y$, except for those edges and vertices involved in the move.

Lemma 5.12 Let $\left(e_{1}, \ldots, e_{n}\right)$ be a monotone segment with $\partial_{0} e_{1}$ minimal. Suppose that a collapse move is performed at $e \in E(X)$, where $G_{e}=G_{\partial_{0} e}$. Then exactly one of the following occurs:

(a) the image of $\left(e_{1}, \ldots, e_{n}\right)$ is monotone with the image of $\partial_{0} e_{1}$ minimal, or

(b) $G_{e} \subsetneq G_{\partial_{1} e}$ and there is a $\gamma \in G$ such that $\partial_{0} \gamma e=\partial_{0} e_{i}$ for some $i$, and $\gamma e \neq e_{i}, \bar{e}_{i-1}$.

Proof Let $v_{i}=\partial_{0} e_{i}$ for $i=1, \ldots, n$ and $v_{n+1}=\partial_{1} e_{n}$. Let $w_{i}$ be the image of $v_{i}$ under the collapse move. Then the image segment $\left[w_{1}, w_{n+1}\right]$ is monotone with $w_{1}$ minimal if and only if $G_{w_{i}} \subseteq G_{w_{i+1}}$ for $i=1, \ldots, n$. Of course, $w_{i}$ and $w_{i+1}$ may be the same vertex for various values of $i$.

(a) $\Rightarrow \neg\left(\right.$ b): Assumption (a) implies that $G_{w_{i}} \subseteq G_{w_{i+1}}$ for each $i$. Now suppose that an element $\gamma \in G$ as in (b) exists. There are two cases. First, if $G_{w_{i+1}}=$ $G_{v_{i+1}}$ then $G_{\partial_{1} \gamma e}=G_{w_{i}} \subseteq G_{v_{i+1}}$. Recall the elementary fact that if $[x, y]$ is any segment with $G_{x} \subseteq G_{y}$ then $G_{x} \subseteq G_{e}$ for every $e \in[x, y]$. Thus, as $\gamma e \in\left[\partial_{1} \gamma e, v_{i+1}\right]$, we have that $G_{\partial_{1} \gamma e} \subseteq G_{\gamma e}$, contradicting (b). The second case is that $G_{w_{i+1}} \supsetneq G_{v_{i+1}}$, meaning that $v_{i+1}=\partial_{0} \delta e$ for some $\delta \in G$, so $G_{w_{i+1}}=G_{\partial_{1} \delta e}$. Notice that $\gamma e \in\left[\partial_{1} \gamma e, \partial_{1} \delta e\right]$, so again we have that $G_{\partial_{1} \gamma e} \subseteq$ $G_{\gamma e}$, contradicting (b).

$\neg$ (b) $\Rightarrow$ (a): If $G_{e}=G_{\partial_{1} e}$ then no vertex stabilizer changes during the collapse move. Thus $G_{w_{i}}=G_{v_{i}}$ for all $i$ and so $G_{w_{i}} \subseteq G_{w_{i+1}}$ for each $i$. Otherwise, if $G_{e} \subsetneq G_{\partial_{1} e}$, then the assumption $\neg(\mathrm{b})$ implies that for every $\gamma \in G$ and every $i \leqslant n$, either $\partial_{0} \gamma e \neq v_{i}$ or $\gamma e=e_{i}$ or $\gamma e=\bar{e}_{i-1}$.

If $v_{i} \notin G \partial_{0} e$ then $G_{w_{i}}=G_{v_{i}}$, so $G_{w_{i}} \subseteq G_{v_{i+1}} \subseteq G_{w_{i+1}}$. If $v_{i}=\partial_{0} \gamma e$ with $i \leqslant n$ then either $\gamma e=e_{i}$ or $\gamma e=\bar{e}_{i-1}$. If $\gamma e=\bar{e}_{i-1}$ then $\partial_{1} \gamma e=v_{i-1}$ and so $G_{w_{i}}=G_{v_{i-1}} \subseteq G_{v_{i+1}} \subseteq G_{w_{i+1}}$. If $\gamma e=e_{i}$ then $\partial_{1} \gamma e=v_{i+1}$, so $G_{w_{i}}=G_{v_{i+1}} \subseteq$ $G_{w_{i+1}}$. Combining all cases we have that $G_{w_{i}} \subseteq G_{w_{i+1}}$ for $i=1, \ldots, n$, and hence the segment $\left[w_{1}, w_{n+1}\right]$ is monotone with $w_{1}$ minimal.

Lemma 5.13 Let $\left(e_{1}, \ldots, e_{n}\right)$ be a segment and suppose that a collapse move is performed at $e \in E(X)$, where $G_{e}=G_{\partial_{0} e}$. Suppose that the image of $\left(e_{1}, \ldots, e_{n}\right)$ is monotone with the image of $\partial_{0} e_{1}$ minimal.

If $\bar{e}_{n} \notin G e$ or $G_{e}=G_{\partial_{1} e}$ then $\left(e_{1}, \ldots, e_{n}\right)$ is monotone with $\partial_{0} e_{1}$ minimal. Otherwise, $\left(e_{1}, \ldots, e_{n-1}\right)$ is monotone with $\partial_{0} e_{1}$ minimal. 
This lemma describes preservation of monotonicity of segments by expansion moves, though it is expressed in terms of a collapse move.

Proof Let $v_{i}=\partial_{0} e_{i}$ for $i=1, \ldots, n$ and $v_{n+1}=\partial_{1} e_{n}$. Let $w_{i}$ be the image of $v_{i}$ under the collapse move. Then $G_{v_{i}} \subseteq G_{w_{i}}$ for each $i$.

For each $i \leqslant n, e_{i}$ and $e_{i+1}$ are not both in $G e$; this would imply that $v_{i+1} \in$ $\left(G \partial_{0} e \cap G \partial_{1} e\right)$, but this set is empty because $e$ admits a collapse move. Similarly, $\bar{e}_{i}$ and $\bar{e}_{i+1}$ are not both in $G e$. If $\bar{e}_{i}, e_{i+1} \in G e$ then there is a $t \in G$ which fixes $\partial_{0} e_{i+1}$ but takes $e_{i+1}$ to $\bar{e}_{i}$. But then $t \in G_{\partial_{0} e_{i+1}}-G_{e_{i+1}}$, contradicting the assumption that $G_{e}=G_{\partial_{0} e}$, and so $\bar{e}_{i}$ and $e_{i+1}$ are not both in $G e$. Finally suppose that $e_{i}, \bar{e}_{i+1} \in G e$, for some $i<n-1$. Notice that since $e_{i}$ collapses to $w_{i+1}, G_{w_{i+1}}=G_{v_{i+1}}$. The previous cases imply that $e_{i+2}, \bar{e}_{i+2} \notin G e$ and so $e_{i+2}$ is not collapsed, and has the same stabilizer after the move. Then monotonicity of $\left[w_{1}, w_{n+1}\right]$ implies that $G_{w_{i+1}}=G_{e_{i+2}}$. Hence $G_{v_{i+1}}=G_{e_{i+2}}$. There is a $t \in G$ fixing $\partial_{1} \bar{e}_{i+1}\left(=v_{i+1}\right)$ and taking $\bar{e}_{i+1}$ to $e_{i}$. Then $t \in G_{e_{i+2}}$, and therefore $t$ fixes $\bar{e}_{i+1}$, as this edge lies between $v_{i+1}$ and $e_{i+2}$. This contradicts $t \bar{e}_{i+1}=e_{i}$, and hence $e_{i}$ and $\bar{e}_{i+1}$ are not both in $G e$.

These facts imply that if $e_{i}$ is collapsed (ie, $e_{i} \in(G e \cup G \bar{e})$ ) then $e_{i+1}$ is not collapsed, unless $i=n-1$ and $e_{n-1}, \bar{e}_{n} \in G e$. Now consider an edge $e_{i}$. If $e_{i}$ is not collapsed then $G_{w_{i}} \subseteq G_{e_{i}}$ by monotonicity, and so $G_{v_{i}} \subseteq G_{w_{i}} \subseteq G_{e_{i}}$.

If $e_{i}$ is collapsed then either $e_{i} \in G e$ or $\bar{e}_{i} \in G e$. In the first case $G_{v_{i}} \subseteq G_{e_{i}}$ holds. So suppose that $\bar{e}_{i} \in G e$. Then $G_{w_{i+1}}=G_{w_{i}}=G_{v_{i}}$, and $G_{e_{i}}=G_{v_{i+1}} \supseteq$ $G_{e_{i+1}}$. Then, assuming that $i<n$, the edge $e_{i+1}$ is not collapsed; the exception $i=n-1, e_{i}, \bar{e}_{i+1} \in G e$, is ruled out because $\bar{e}_{i} \in G e$. Thus after the collapse $e_{i+1}$ is present, with stabilizer $G_{e_{i+1}}$, and $G_{w_{i+1}} \subseteq G_{e_{i+1}}$ by monotonicity. Hence $G_{v_{i}} \subseteq G_{e_{i+1}} \subseteq G_{e_{i}}$.

Thus we have shown that $G_{v_{i}} \subseteq G_{e_{i}}$ for all $i<n$, and for $i=n$ if $\bar{e}_{n} \notin G e$. If $\bar{e}_{n} \in G e$ and $G_{e}=G_{\partial_{1} e}$ then $G_{v_{n}}=G_{e_{n}}$ by equivariance.

Lemma 5.14 Let $X$ be a telescoping of $Z$, with $Z$ strongly slide-free. Let $[V, W] \subseteq X$ be a $Z$-telescope. Suppose that $e$ is a collapsible edge of $X$, with $G e \cap[V, W]=\varnothing$. Then one of the following occurs:

(a) $G \partial_{0} e \cap[V, W]=\varnothing$, or

(b) $G \partial_{1} e \cap[V, W]=\varnothing$, or

(c) $G \partial_{0} e$ and $G \partial_{1} e$ each meet $[V, W]$ in one endpoint, and the corresponding elements of $G e$ are in $X_{V}$ and $X_{W}$. 
Proof Recall that collapsibility of $e$ means that $G_{e}=G_{\partial_{0} e}$ and $G \partial_{0} e \cap G \partial_{1} e=$ $\varnothing$. Suppose that (a) and (b) do not hold, so that $[V, W]$ meets both $G \partial_{0} e$ and $G \partial_{1} e$. Relabeling if necessary, we have $\partial_{0} e \in[V, W]$ and $\partial_{1} \gamma e \in[V, W]$ for some $\gamma \in G$. Note that by Lemma 5.6 the endpoints of $e$ are in $X_{G}$, and hence $e \in X_{G}$. For every $U \neq V, W$ in $M(X), X_{U}$ does not meet $[V, W]$ and therefore $e \notin X_{U}$. If $e \in X_{V}$ then $\partial_{0} e$ is the endpoint $[V, W] \cap X_{V}$. The edge $\gamma e$ is in $X_{\left(V^{\gamma}\right)}$, and since $\partial_{1} \gamma e \in[V, W]$ we must have $X_{\left(V^{\gamma}\right)}=X_{W}$. If $G \partial_{0} e$ and $G \partial_{1} e$ do not meet the interior of $[V, W]$ then condition (c) now holds. The case $e \in X_{W}$ is similar.

Otherwise, we can assume that $e, \gamma e \notin X_{U}$ for all $U \in M(X)$. Then Lemma 5.6 implies that $e$ is in another $Z$-telescope, which has a common endpoint with $[V, W]$ by Lemma 5.7 .

There are now two cases. Suppose first that the $Z$-telescope containing $e$ has the form $\left[V, W^{\prime}\right]$. Then $\gamma e$ is in the $Z$-telescope $\gamma\left(\left[V, W^{\prime}\right]\right)$, so Lemma 5.7 implies that $\{V, W\} \cap\left\{V^{\gamma},\left(W^{\prime}\right)^{\gamma}\right\} \neq \varnothing$. Note that $e$ separates $X_{V}$ from $\partial_{1} e$, and so $\gamma e$ separates $X_{\left(V^{\gamma}\right)}$ from $\partial_{1} \gamma e \in[V, W]$. Hence $V^{\gamma} \neq V, W$ and so $\{V, W\} \cap\left\{V^{\gamma},\left(W^{\prime}\right)^{\gamma}\right\}=\left\{\left(W^{\prime}\right)^{\gamma}\right\}$. Thus $\left(W^{\prime}\right)^{\gamma}=V$ or $\left(W^{\prime}\right)^{\gamma}=W$.

The $Z$-telescope $\left[V, W^{\prime}\right]$ is separated by $e$ into two segments, $\left[V, \partial_{0} e\right]$ and $\left[\partial_{1} e, W^{\prime}\right]$. The first is contained in the $Z$-telescope $[V, W]$. The second is mapped by $\gamma$ either to $\left[\partial_{1} \gamma e, V\right] \subseteq[V, W]$ or to $\left[\partial_{1} \gamma e, W\right] \subseteq[V, W]$, and hence $\left[\partial_{1} e, W^{\prime}\right]$ is contained in the $Z$-telescope $\gamma^{-1}([V, W])$. Therefore $\{e, \bar{e}\}$ is the only geometric edge of $\left[V, W^{\prime}\right]$ that is not contained in another $Z$-telescope. It follows, from property 5.5(iii), that $e$ is a $Z$-minimal edge for $\left[V, W^{\prime}\right]$. But the vertex $\partial_{0} e$ is in two $Z$-telescopes, contradicting Lemma 5.11 .

The second case, when the $Z$-telescope containing $e$ has the form $\left[V^{\prime}, W\right]$, yields a similar contradiction.

Proposition 5.15 Let $Z$ be a strongly slide-free $G$-tree, and suppose that $X$ is a telescoping of $Z$. Let $Y$ be obtained from $X$ by an elementary move. Then $Y$ is a telescoping of $Z$.

Proof First note that elementary moves do not change the set of maximal stabilizers, and that $Z$ possesses maximal stabilizers, because it is proper. Then $M(X)=M(Z)$ and $M(Y)=M(X)$. To avoid confusion, we will decorate segments with subscripts to indicate which tree they are in.

Step 1A Invariance of 5.5(i) and 5.5(iii) under collapses Let $[V, W]_{X}$ be a $Z$-telescope. Suppose the edge $e$ is collapsed, where $G_{e}=G_{\partial_{0} e}$. First we arrange that the $Z$-minimal edge $e_{V W}$ is not contained in $G e \cup G \bar{e}$. 
If $e_{V W} \in(G e \cup G \bar{e})$, then we have $e_{V W}=\gamma e$ for some $\gamma \in G$ (replacing $e_{V W}$ by its inverse if necessary). Then $G_{\partial_{0} e_{V W}}=G_{e_{V W}}=V \cap W$, and so $\partial_{0} e_{V W}$ is not an endpoint of $[V, W]_{X}$. Let $e^{\prime}$ be the unique edge in $\left(E_{0}\left(\partial_{0} e_{V W}\right) \cap\right.$ $\left.[V, W]_{X}\right)-\left\{e_{V W}\right\}$. Since $[V, W]_{X}$ is a telescope, $G_{e^{\prime}}=G_{e_{V W}}$, and so $e^{\prime}$ is a $(V, W)$-minimal edge. If $e^{\prime} \notin[v, w]$ then $\partial_{0} e_{V W}$ is an endpoint of $[v, w]$, which implies that this telescope is not proper. However, $X$ is a proper telescoping of $Z$ by Lemma 5.10, so we conclude that $e^{\prime} \in[v, w]$. Note also that $e^{\prime}$ is not contained in any other $Z$-telescope of $X$, for then $\partial_{0} e_{V W}$ would be in two $Z$ telescopes, contradicting Lemma 5.11. Finally we note that $e^{\prime} \notin(G e \cup G \bar{e})$. If $e^{\prime} \in G \bar{e}$ then $\partial_{0} e_{V W} \in G \partial_{0} e \cap G \partial_{1} e$, but this set is empty as $e$ is collapsible. If $e^{\prime} \in G e$ then $\delta e^{\prime}=e_{V W}$ for some $\delta \in G$, but then $\delta \in\left(G_{\partial_{0} e_{V W}}-G_{e_{V W}}\right)=\varnothing$.

Thus, replacing $e_{V W}$ with $e^{\prime}$ if necessary, there is a $Z$-minimal edge $e_{V W} \notin$ $(G e \cup G \bar{e})$. Then $e_{V W}$ is present after the collapse move. We can orient $e_{V W}$ so that $\partial_{0} e_{V W}$ separates $X_{V}$ from $e_{V W}$. Applying Lemma 5.12 to the monotone segments $\left[V, \partial_{0} e_{V W}\right]_{X}$ and $\left[\partial_{1} e_{V W}, W\right]_{X}$, we find that $[V, W]_{Y}$ is a telescope with $(V, W)$-minimal edge $e_{V W}$, unless $G_{e} \subsetneq G_{\partial_{1} e}$ and $\partial_{0} \gamma e$ is in the interior of $[V, W]_{X}$ for some $\gamma \in G$, and $\gamma e \notin[V, W]_{X}$.

If this occurs, then note that $[V, W]_{X} \subseteq X_{G}$ by Lemma 5.6, and so $\gamma e \in X_{G}$ by Lemma 5.3. Also, $\gamma e \notin X_{U}$ for all $U \in M(X)$, since no $X_{U}$ meets the interior of $[V, W]_{X}$. Hence, by Lemma 5.6, $\gamma e$ is contained in a $Z$-telescope. Lemma 5.7 implies that this telescope has a common endpoint with $[V, W]_{X}$. Suppose the telescope is $\left[V, W^{\prime}\right]_{X}$. Note that the subsegment $\left[\partial_{1} \gamma e, W^{\prime}\right] \subseteq\left[V, W^{\prime}\right]_{X}$ cannot contain a $\left(V, W^{\prime}\right)$-minimal edge, because $G_{\gamma e} \subsetneq G_{\partial_{1} \gamma e}$. The segment $\left[V, \partial_{0} \gamma e\right]$ is the intersection of $Z$-telescopes $[V, W]_{X}$ and $\left[V, W^{\prime}\right]_{X}$, and hence it does not contain a $Z$-minimal edge. Thus the $\left(V, W^{\prime}\right)$-minimal edge promised by property 5.5 (iii) can only be $\gamma e$ or $\gamma \bar{e}$. Hence, $\gamma e$ is a $Z$-minimal edge for $\left[V, W^{\prime}\right]_{X}$ (and so is $\gamma \bar{e}$ ). Now $\partial_{0} \gamma e$ is in two $Z$-telescopes, contradicting Lemma 5.11. A similar contradiction ensues if the $Z$-telescope containing $\gamma e$ has the form $\left[V^{\prime}, W\right]_{X}$.

Therefore $[V, W]_{Y}$ is a telescope with $(V, W)$-minimal edge $e_{V W}$. It is clear that $e_{V W}$ is not contained in any other $Z$-telescope of $Y$, since every $\left[V^{\prime}, W^{\prime}\right]_{Y}$ is the image of $\left[V^{\prime}, W^{\prime}\right]_{X}$, and this property holds in $X$. Lastly, we need to know that $e_{V W}$ is contained in the injective sub-segment $\left[v^{\prime}, w^{\prime}\right]_{Y} \subseteq[V, W]_{Y}$ given by property 5.5(ii) of $Y$. In Step $2 \mathrm{~A}$ we show that $Y$ indeed satisfies $5.5(\mathrm{ii})$, and that $\left[v^{\prime}, w^{\prime}\right]_{Y}$ is the image of $[v, w]_{X}$. Therefore $e_{V W} \in\left[v^{\prime}, w^{\prime}\right]_{Y}$.

Step 1B Invariance of 5.5(i) under expansions Suppose that an expansion move is performed, creating $e$ with $G_{e}=G_{\partial_{0} e}$. By Lemma 5.13, $[V, W]_{Y}$ is a telescope, unless the following conditions hold: $\gamma e \in[V, W]_{Y}$ for some $\gamma \in G$ 
with $\partial_{0} \gamma e$ equal to one of the endpoints of $[V, W]_{Y}$, and $G_{e} \subsetneq G_{\partial_{1} e}$. But then $G_{\partial_{0} \gamma e}=G_{\gamma e} \subsetneq G_{\partial_{1} \gamma e}$, which contradicts maximality of $V$ or $W$ (one of which is equal to $\left.G_{\partial_{0} \gamma e}\right)$.

Step 2A Invariance of 5.5(ii) under collapses Let $[V, W]_{X}$ be a $Z$-telescope, and suppose that the edge $e$ is collapsed, where $G_{e}=G_{\partial_{0} e}$. The image of $[V, W]_{X}$ in $Y$ is $[V, W]_{Y}$, which is a $Z$-telescope by Step $1 \mathrm{~A}$. Let $v^{\prime}$ and $w^{\prime}$ be the images of $v$ and $w$ respectively. If $t\left([V, v]_{X}\right)=[W, w]_{X}$ then also $t\left(\left[V, v^{\prime}\right]_{Y}\right)=\left[W, w^{\prime}\right]_{Y}$. Note that $\left[V, w^{\prime}\right]_{Y}$ and $\left[v^{\prime}, W\right]_{Y}$ are telescopes, since they are nontrivial sub-segments of the telescope $[V, W]_{Y}$. Now, to establish 5.5 (ii) for $Y$, it remains to show that $\left[V, w^{\prime}\right]_{Y}$ and $\left[v^{\prime}, W\right]_{Y}$ are injective (we are not required to show that $\left[v^{\prime}, w^{\prime}\right]$ is proper).

If $\left[V, w^{\prime}\right]_{Y}$ is not injective then two of its interior vertices are in the same orbit. The preimages in $[V, w]_{X}$ of these vertices must be incident to opposite ends of the geometric edge orbit $G\{e, \bar{e}\}$. By relabeling if necessary, we can arrange that $\partial_{0} e$ and $\partial_{1} \gamma e$ are in the interior of $[V, w]_{X}$ for some $\gamma \in G$. Injectivity of $[V, w]_{X}$ implies that if $\delta e \in[V, w]_{X}$ for some $\delta \in G$, then the endpoints of $\delta e$ are among the vertices $\partial_{0} e, \partial_{1} \gamma e, w$, and the endpoint of $[V, w]_{X}$ in $X_{V}$. But $\delta e$ does not join $\partial_{0} e$ to $\partial_{1} \gamma e$ because these vertices have separate images in $Y$. Also $\delta e$ does not join an endpoint of $[V, w]_{X}$ to one of $\partial_{0} e, \partial_{1} \gamma e$, because the images of these vertices are in the interior of $\left[V, w^{\prime}\right]_{Y}$. Therefore $G e \cap[V, w]_{X}=\varnothing$. By property 5.5(ii) we have $G e \cap[w, W]_{X}=\varnothing$, and so $G e \cap[V, W]_{X}=\varnothing$. We now obtain a contradiction by applying Lemma 5.14, since none of its three conclusions holds. Thus, $\left[V, w^{\prime}\right]_{Y}$ is injective. Similarly, $\left[v^{\prime}, W\right]_{Y}$ is injective.

Step 2B Invariance of 5.5(ii) under expansions Suppose that an expansion move is performed at $u$, creating the edge $e$ with $G_{e}=G_{\partial_{0} e}$. Consider the intersection of $[V, W]_{Y}$ with $G e \cup G \bar{e}$. First we observe that no two adjacent edges of $[V, W]_{Y}$ are in $G e \cup G \bar{e}$. For if this occurs, then the two edges cannot be coherently oriented. Their common endpoint would be in $G \partial_{0} e \cap G \partial_{1} e$, but this intersection is empty because $e$ is collapsible. Also, if the two edges were incoherently oriented, then they would be related by an element of $G$ fixing their common endpoint. This element would violate the telescope property of $[V, W]_{Y}$, which holds by Step 1B.

Thus, no two geometric edges in $[V, W]_{Y} \cap(G e \cup G \bar{e})$ collapse to the same vertex of $X$. The telescope $[V, W]_{Y}$ is now obtained from $[V, W]_{X}$ by replacing each of various vertices in $G u \cap[V, W]_{X}$ by a single geometric edge in $G\{e, \bar{e}\}$ (vertices are omitted if the corresponding elements of $G\{e, \bar{e}\}$ are not in $\left.[V, W]_{Y}\right)$. Note that property 5.5(ii) implies that $[V, W]_{X}$ contains at most two vertices of $G u$, 
and if there are two, then they are in $[V, v]$ and $[W, w]$ respectively, and are related by $t$.

Suppose that $\gamma u$ is in the interior of $[V, v]_{X}$, and suppose that $\gamma u$ expands to $\gamma e \in[V, v]_{Y}$. Let $e_{1}, e_{2} \in E_{0}(\gamma u)$ be the two neighboring edges of $[V, v]_{X}$. Then $e_{1}$ and $e_{2}$ are separated by $\gamma e$ in $Y$, and $t e_{1}$ and $t e_{2}$ are in $[V, W]_{Y}$, separated by $t \gamma e$. Hence $t \gamma e \in[w, W]_{Y}$. Similarly if $\gamma u$ is the endpoint $[V, W]_{X} \cap X_{V}$ and $\gamma e \in[V, W]_{Y}$, then $t \gamma e \in[V, W]_{Y}$. In each of these cases, $G u$ does not meet $[v, w]$ by property 5.5 (ii), and so $[v, w]$ is unchanged during the move, and hence $[V, w]_{Y}$ and $[v, W]_{Y}$ are injective. Also, the relation $t\left([V, v]_{Y}\right)=[w, W]_{Y}$ holds.

Next suppose that $v=\gamma u$ for some $\gamma \in G$. If $w \notin G u$ then $[v, w]=[V, W]_{X}$ and $[V, W]_{Y}$ is injective. Otherwise, $w=t v$ for some $t$, and $t([V, v])=[W, w]$. Recall that 5.5(ii) implies that $G u$ meets $[V, W]_{X}$ in $v$ and $w$ only. Then $[V, W]_{Y}$ meets $G\{e, \bar{e}\}$ in zero, one, or two geometric edges. If $[V, W]_{Y} \cap$ $G\{e, \bar{e}\}=\varnothing$, let $v^{\prime}$ and $w^{\prime}$ be the vertices of $[V, W]_{Y}$ that are incident to $G\{e, \bar{e}\}$, and which map to $v$ and $w$ respectively under the collapse move from $Y$ to $X$. Then $\left[V, w^{\prime}\right]_{Y}$ and $\left[v^{\prime}, W\right]_{Y}$ are injective and $t\left(\left[V, v^{\prime}\right]_{Y}\right)=\left[W, w^{\prime}\right]_{Y}$. If $[V, W]_{Y}$ meets $G e$ in one edge $\gamma e$ which collapses to $v \in X$, let $v^{\prime}$ be the endpoint of $\gamma e$ closest to $Y_{V}$. Let $w^{\prime} \in[V, W]_{Y}$ be the vertex corresponding to $w \in[V, W]_{X}$. Then $t\left(\left[V, v^{\prime}\right]_{Y}\right)=\left[W, w^{\prime}\right]_{Y}$, and $\left[V, w^{\prime}\right]_{Y}$ and $\left[v^{\prime}, W\right]_{Y}$ are injective.

If $[V, W]_{Y}$ contains two edges of $G e$, then call the edges $\gamma e$ and $\delta e$, where $\gamma e$ collapses to $\gamma u=v$ and $\delta e$ collapses to $t \gamma u=w$. Let $v^{\prime \prime}$ and $w^{\prime \prime}$ be the endpoints of $[\gamma e, \delta e]$. Then $\left[V, v^{\prime \prime}\right]_{Y}$ and $\left[W, w^{\prime \prime}\right]_{Y}$ map bijectively to $[V, v]_{X}$ and $[W, w]_{X}$ respectively under the collapse from $Y$ to $X$. Note that $t\left(\left[V, v^{\prime \prime}\right]_{Y}\right)=$ $\left[W, w^{\prime \prime}\right]_{Y}$.

We have that $v^{\prime \prime}=\partial_{i} \gamma e$ for $i=0$ or 1 . Then $t v^{\prime \prime}=w^{\prime \prime}$ implies $\partial_{i} t \gamma e=w^{\prime \prime}$. Now if $\partial_{(1-i)} \delta e=w^{\prime \prime}$, then $w^{\prime \prime} \in\left(G \partial_{0} e \cap G \partial_{1} e\right)$, contradicting the fact that $e$ is collapsible. Hence $\partial_{i} \delta e=w^{\prime \prime}=\partial_{i} t \gamma e$. This implies that $\delta \gamma^{-1} t^{-1}$ fixes $w^{\prime \prime}$, because $\delta \gamma^{-1} t^{-1}(t \gamma e)=\delta e$. Since $\left[w^{\prime \prime}, W\right]_{Y}$ is monotone with $w^{\prime \prime}$ minimal, $\delta \gamma^{-1} t^{-1}$ also fixes all of $\left[w^{\prime \prime}, W\right]_{Y}$. Therefore $\delta \gamma^{-1}([V, \gamma e])=[W, \delta e]$. Setting $v^{\prime}=\partial_{(1-i)} \gamma e$ and $w^{\prime}=\partial_{(1-i)} \delta e$, we now have that the injective segment $\left[v^{\prime}, w^{\prime}\right]_{Y} \subseteq[V, W]_{Y}$ is the sub-telescope required by property 5.5 (ii), where the element $\delta \gamma^{-1}$ has taken the place of $t$.

Lastly suppose that $G u$ meets the interior of $[v, w]$. Then 5.5(ii) implies that $G u$ meets $[V, W]_{X}$ in no other point. Then $[V, w]_{Y}$ and $[v, W]_{Y}$ are injective and so 5.5(ii) is satisfied by $Y$. 
Step 3 Invariance of 5.5(iii) under expansions Suppose that an expansion move is performed. Let $e_{V W}$ be a $Z$-minimal edge for $[V, W]_{X}$. In Step 1B we showed that $e_{V W}$ is $(V, W)$-minimal for $[V, W]_{Y}$. It is contained in the segment $\left[v^{\prime}, w^{\prime}\right]_{Y} \subseteq[V, W]_{Y}$, according to the description of $\left[v^{\prime}, w^{\prime}\right]_{Y}$ obtained in Step 2B. Also $e_{V W}$ is not contained in another $Z$-telescope after the move, and so it is $Z$-minimal.

Definition 5.16 Let $X$ be a $G$-tree which reduces to $Z$. We say that $X$ is uniquely reducible to $Z$ if every maximal sequence of collapse moves performed on $X$ results in the $G$-tree $Z$.

The following result includes Theorem 1.2 of the Introduction.

Theorem 5.17 Let $X$ be a strongly slide-free $G$-tree, and let $Y$ be obtained from $X$ by an elementary deformation. If $Y$ is proper, or cocompact and reduced, then there is a unique isomorphism of $G$-trees $X \rightarrow Y$. Otherwise $Y$ is a proper telescoping of $X$. If $Y$ is cocompact then it is uniquely reducible to $X$.

Proof Proposition 5.15 and Lemma 5.10 together imply that the property of being a proper telescoping of $Z$, when $Z$ is strongly slide-free, is invariant under elementary deformations. Then since $X$ is a proper telescoping of itself, $Y$ is a proper telescoping of $X$. If $Y$ is proper then $Y \cong X$ as remarked earlier. Uniqueness of the isomorphism follows from the fact that the only equivariant self map of a proper $G$-tree is the identity. To prove this fact, let $\phi: Z \rightarrow Z$ be equivariant. If $Z$ is proper then the stabilizer of a vertex does not fix any other vertex. Since equivariance requires $G_{\phi(z)} \subseteq G_{z}$ for all $z$, the map is the identity.

For the last conclusion of the theorem, let $\widehat{Y}$ be obtained from $Y$ by a maximal sequence of collapse moves. Then $\widehat{Y}$ is related to $X$ by a deformation, so it is a proper telescoping of $X$ by 5.15 and 5.10. Since $\widehat{Y}$ is reduced, every $X-$ telescope in $\widehat{Y}$ has length 1 , by properties 5.5(i) and 5.5(ii). Thus $\widehat{Y} \cong X$. The case when $Y$ is cocompact and reduced follows from the statement just proved.

\section{Rigidity}

In this section we combine Theorems 4.2 and 5.17 to generalize the rigidity theorem of Bass and Lubotzky given in [4]. This result is Corollary 6.5. The 
graph of groups formulation of this result then yields Corollary 1.4 of the Introduction. We also apply the theory to generalized Baumslag-Solitar trees (defined below), in Corollary 6.10.

First we have the following immediate consequence of Theorems 4.2 and 5.17. It includes Corollary 1.3 of the Introduction.

Theorem 6.1 Let $G$ be a group. Let $X$ and $Y$ be cocompact $G$-trees with the same elliptic subgroups. Suppose that $X$ is strongly slide-free. Then $Y$ is a proper telescoping of $X$, and hence is uniquely reducible to $X$. If in addition $Y$ is reduced, then there is a unique isomorphism of $G$-trees $X \rightarrow Y$.

If one imposes fixed point properties on the vertex stabilizers of a $G$-tree, then the set of elliptic subgroups may be uniquely determined. Or, this conclusion may hold for other reasons. The rest of the results in this section concern such phenomena.

Proposition 6.2 Let $X$ and $Y$ be proper $G$-trees which define the same partition of $G$ into elliptic and hyperbolic elements. Suppose in addition that $X$ is locally finite. Then every vertex stabilizer of $X$ has a fixed point in $Y$.

Proof Call a subgroup of $G$ weakly elliptic if its elements are all elliptic. The vertex stabilizers of $X$ and $Y$ are maximal weakly elliptic subgroups, by properness (and the Hyperbolic Segment Condition).

Let $H=G_{x}$ be a vertex stabilizer of $X$ and consider its action on $Y$. By Proposition 2.6, $H$ fixes either a vertex or an end of $Y$. Suppose $H$ fixes $\varepsilon \in \partial Y$. Let $\mathscr{E}$ be the set of elliptic elements of $G$; then $H \subseteq\left(G_{\varepsilon} \cap \mathscr{E}\right)$. Note that $G_{\varepsilon} \cap \mathscr{E}$ is a subgroup (and is weakly elliptic), and hence $H=G_{\varepsilon} \cap \mathscr{E}$ by maximality.

Suppose first that $\varepsilon$ is rational, meaning that $G_{\varepsilon}$ contains a hyperbolic element $\gamma$ (ie, $\varepsilon$ is an endpoint of some hyperbolic axis). Then $\gamma H \gamma^{-1}$ is maximal weakly elliptic and is also contained in $G_{\varepsilon} \cap \mathscr{E}$, hence is equal to $H$. Since $\gamma$ is hyperbolic, the subgroups $H$ and $\gamma H \gamma^{-1}$ are the stabilizers of different vertices of $X$, contradicting properness.

Next assume that $\varepsilon$ is irrational, so that $G_{\varepsilon} \subseteq \mathscr{E}$, and hence $H=G_{\varepsilon}$. Let $\left(y_{i}\right)_{i \geqslant 0}$ be any sequence of vertices of $Y$ tending monotonically to $\varepsilon$. Then $G_{\varepsilon}$ is the increasing union $\bigcup_{i \geqslant 0}\left(G_{\varepsilon}\right)_{y_{i}}$. We can assume that the inclusions $\left(G_{\varepsilon}\right)_{y_{i}} \subseteq\left(G_{\varepsilon}\right)_{y_{i+1}}$ are strict infinitely often, for otherwise $G_{\varepsilon}$ would fix a vertex of $Y$. Each $\left(G_{\varepsilon}\right)_{y_{i}}=\left(G_{y_{i}}\right)_{\varepsilon}$ is equal to the intersection $\bigcap_{j \geqslant i} G_{y_{j}}$, and therefore

$$
G_{\varepsilon}=\bigcup_{i \geqslant 0} \bigcap_{j \geqslant i} G_{y_{j}} .
$$


Now we ask whether a vertex stabilizer of $X$ can have this structure. Each stabilizer $G_{y_{i}}$ fixes either a vertex or an end of $X$, and in the latter case the end must be irrational as above. Then, by maximality of $G_{y_{i}}$, it must be equal to the stabilizer of that vertex or end. Thus we have $G_{y_{i}}=G_{z_{i}}$ for some $z_{i} \in V(X) \cup \partial X$. Note that $\left\{z_{i}\right\}$ is infinite, for otherwise only finitely many of the inclusions $\left(G_{\varepsilon}\right)_{y_{i}} \subseteq\left(G_{\varepsilon}\right)_{y_{i+1}}$ would be strict.

Let $B_{1}(x)$ denote the ball of radius 1 at $x$. As $X$ is locally finite, $B_{1}(x)$ separates $X \cup \partial X$ into finitely many connected components, one of which meets $\left\{z_{i}\right\}$ in an infinite set. Thus, by passing to a subsequence we can arrange that $\left\{z_{i}\right\}$ is contained in a connected component of $(X \cup \partial X)-B_{1}(x)$. Note that equation (6.3) remains valid after passing to any subsequence. Now consider the subtree spanned by $\left\{z_{i}\right\} . B_{1}(x)$ is disjoint from this subtree, so there is a vertex $x^{\prime}$ separating $x$ from every $z_{i}$. Then $G_{x} \cap G_{z_{i}} \subseteq G_{x^{\prime}}$ for all $i$, hence $G_{x} \cap \bigcap_{j \geqslant i} G_{z_{j}} \subseteq G_{x^{\prime}}$ for all $j$. But $G_{x} \cap \bigcap_{j \geqslant i} G_{z_{j}}=G_{\varepsilon} \cap \bigcap_{j \geqslant i} G_{z_{j}}=$ $\bigcap_{j \geqslant i} G_{z_{j}}=\bigcap_{j \geqslant i} G_{y_{j}}$, so (6.3) yields $G_{\varepsilon} \subseteq G_{x^{\prime}}$. This contradicts properness of $X$ as $G_{\varepsilon}=G_{x}$.

Proposition 6.4 Let $X$ and $Y$ be cocompact $G$-trees which define the same partition of $G$ into elliptic and hyperbolic elements. Suppose that $Y$ is proper, and that every vertex stabilizer of $X$ has a fixed point in $Y$. Then $X$ and $Y$ have the same elliptic subgroups.

Proof In fact, we show directly that $X$ and $Y$ are related by a deformation, which implies the conclusion by Remark 3.5. The proof is nearly the same as the proof of Theorem 4.2. We can assume without loss of generality that $X$ is reduced, by performing collapse moves. By Proposition 4.16 there exist a $G$-tree $X^{\prime}$ obtained from $X$ by subdivision and an equivariant morphism $\phi: X^{\prime} \rightarrow Y$. This morphism is surjective because $Y$ is proper and hence minimal. Thus, by Proposition 4.7, it factors as $\phi=\psi \rho$ where $\rho: X^{\prime} \rightarrow Z$ is a finite composition of folds and $\psi: Z \rightarrow Y$ induces an isomorphism of quotient graphs.

Since $Y$ is proper, Proposition 4.11(a) implies that $\psi$ is an isomorphism. Thus, $\phi$ is a finite composition of folds. Each individual fold is equivariant and therefore it preserves ellipticity of elements of $G$. Since their composition $\phi$ preserves hyperbolicity, each fold must preserve hyperbolicity as well. Therefore Proposition 3.16 applies to each fold and $X^{\prime}$ and $Y$ are related by an elementary deformation. Then $X$ and $Y$ are related by a deformation. 
Corollary 6.5 Let $G$ be a group. Let $X$ be a strongly slide-free $G$-tree and $Y$ a proper $G$-tree, both cocompact. Suppose that all vertex stabilizers are unsplittable. If either

(a) one of the trees has (FA) vertex stabilizers, or

(b) one of the trees is locally finite,

then there is a unique isomorphism of $G$-trees $X \rightarrow Y$.

Proof The assumptions imply that $X$ and $Y$ define the same partition of $G$ into elliptic and hyperbolic elements. By Theorem 6.1 it suffices to show that $X$ and $Y$ have the same elliptic subgroups. Note that both trees are proper by Remark 5.2. Exchanging names if necessary, assume that $X$ is the tree referred to in condition (a) or (b). If (a) holds then $X$ and $Y$ have the same elliptic subgroups by Proposition 6.4. If (b) holds then Propositions 6.2 and 6.4 together yield the same conclusion.

Question 6.6 Let $X$ and $Y$ be proper cocompact $G$-trees which define the same partition of $G$ into elliptic and hyperbolic elements. Do $X$ and $Y$ have the same elliptic subgroups? If so, then the hypotheses (a) and (b) of Corollary 6.5 could be dropped.

Corollary 1.4 of the Introduction is a special case of the following result, which is a graph of groups interpretation of Corollary 6.5.

Corollary 6.7 Let $\mathbf{A}=(A, \mathscr{A}, \alpha)$ and $\mathbf{B}=(B, \mathscr{B}, \beta)$ be graphs of groups with finite underlying graphs and unsplittable vertex groups. Suppose that $\mathbf{A}$ is strongly slide-free and $\mathbf{B}$ is proper, and that either:

(a) one of the graphs of groups has (FA) vertex groups, or

(b) one of the graphs of groups has finite index edge-to-vertex inclusions.

Let $a_{0}$ and $b_{0}$ be basepoints in $A$ and $B$ respectively, and suppose that there is an isomorphism $\psi: \pi_{1}\left(\mathbf{A}, a_{0}\right) \rightarrow \pi_{1}\left(\mathbf{B}, b_{0}\right)$. Then there exist an isomorphism $\Phi=(\phi,(\gamma)): \mathbf{A} \rightarrow \mathbf{B}$ and an element $g \in \pi\left[\phi\left(a_{0}\right), b_{0}\right] \subseteq \pi(\mathbf{B})$ such that $\psi$ factors as

$$
\pi_{1}\left(\mathbf{A}, a_{0}\right) \stackrel{\Phi_{a_{0}}}{\longrightarrow} \pi_{1}\left(\mathbf{B}, \phi\left(a_{0}\right)\right) \stackrel{\operatorname{ad}(g)}{\longrightarrow} \pi_{1}\left(\mathbf{B}, b_{0}\right) .
$$

In particular, $\mathbf{A}$ and $\mathbf{B}$ are isomorphic as graphs of groups.

Recall that our notion of isomorphism for graphs of groups is taken from [1, Section 2], and is more inclusive than the naive definition (cf Corollary 4.5). For the additional notation and definitions we refer to [1]. The conclusion and proof of 6.7 are the same as those of Corollary 4.5 in [4]. 
Proof Let $G=\pi_{1}\left(\mathbf{A}, a_{0}\right), X=\left(\widetilde{\mathbf{A}, a_{0}}\right)$, and $Y=\left(\widetilde{\mathbf{B}, b_{0}}\right)$. Then $X$ and $Y$ are $G$-trees (via $\psi$ in the case of $Y$ ), and they satisfy the hypotheses of Corollary 6.5. Hence there is an isomorphism of $G$-trees $\phi: X \rightarrow Y$. By $[1,4.2-4.5]$ there is an isomorphism $\Phi=(\phi,(\gamma)): \mathbf{A} \rightarrow \mathbf{B}$. The element $g \in \pi\left[\phi\left(a_{0}\right), b_{0}\right]$ and factorization $\psi=\operatorname{ad}(g) \circ \Phi_{a_{0}}$ exist by [2, Section 3 and Theorem 4.1].

It turns out that a more restrictive variant of Corollary 6.5 can be proved without using the deformation theory of section 4 . This version does not assume cocompactness. Following [4], a $G$-tree is strict if no edge stabilizer contains the stabilizer of another geometric edge or vertex. Strict $G$-trees are strongly slide-free, but the converse does not hold. For example, a generalized BaumslagSolitar tree that is not a point (see definition 6.9 below) cannot be strict, but it can easily be slide-free or strongly slide-free.

Theorem 6.8 Let $G$ be a group and let $X$ and $Y$ be strict $G$-trees. If all vertex stabilizers have property (FA) then there is a unique isomorphism of $G$-trees $X \rightarrow Y$.

Proof Since both trees have (FA) vertex stabilizers, they have the same elliptic subgroups. Then, as each tree is proper, the vertex stabilizers in each tree are precisely the maximal elliptic subgroups. Thus the trees have the same vertex stabilizers. By Proposition (3.5)(d) of [4] the trees are canonically isomorphic. It is here where strictness of both trees is required.

The essential content of the proposition cited above is the following. If $X$ is a strict $G$-tree then it is proper, and hence vertices correspond bijectively with their stabilizers. Then, using strictness, one finds that two vertices bound an edge if and only if the intersection of their stabilizers is maximal among pairwise intersections of vertex stabilizers. Thus $X$ is intrinsically determined by its set of vertex stabilizers. The property of strictness seems to be the most general condition under which this conclusion holds.

Definition 6.9 A generalized Baumslag-Solitar tree is a $G$-tree in which all edge and vertex stabilizers are infinite cyclic. The groups $G$ which arise are called generalized Baumslag-Solitar groups. Examples include the classical Baumslag-Solitar groups, torus knot groups, and finite index subgroups of these groups.

Note that the preceding results do not apply to generalized Baumslag-Solitar trees because $\mathbb{Z}$ is not unsplittable. The results of [4] also do not apply. However, the elliptic subgroups of generalized Baumslag-Solitar groups are easily determined, and we have the following result. 
Corollary 6.10 Let $X$ and $Y$ be cocompact generalized Baumslag-Solitar trees with group $G$. Assume that each tree has a minimal subtree not contained in a line. Then there is an elementary deformation relating $X$ to $Y$. If $X$ is strongly slide-free, then $Y$ is uniquely reducible to $X$. If in addition $Y$ is reduced, then there is a unique isomorphism $X \rightarrow Y$.

The assumption on minimal subtrees is directly analogous to the Kleinian groups property of being non-elementary: that the limit set has more than two points. If a $G$-tree $X$ has a minimal subtree, then the boundary of this subtree is the limit set of $X$. In this way the two properties are seen to coincide. If a cocompact generalized Baumslag-Solitar tree does not have this property then the group is isomorphic to $\mathbb{Z}, \mathbb{Z} \times \mathbb{Z}$, or the Klein bottle group.

Proof First note that $G$ is torsion free: every hyperbolic element has infinite order and every elliptic element is contained in an infinite cyclic subgroup. Also note that for any $\gamma \in G$ and $i \neq 0$, the element $\gamma^{i}$ is hyperbolic if and only if $\gamma$ is, and when this occurs they have the same axis.

We will say that two subgroups $H, H^{\prime} \subseteq G$ are commensurable if $H \cap H^{\prime}$ has finite index in both $H$ and $H^{\prime}$. Commensurability is an equivalence relation on the subgroups of $G$. We say that two nontrivial elements $\gamma, \delta \in G$ are commensurable if the infinite cyclic subgroups they generate are commensurable. This occurs if and only if $\gamma^{i}=\delta^{j}$ for some $i, j \neq 0$. Thus, commensurable hyperbolic elements have the same axis.

In a generalized Baumslag-Solitar tree every edge stabilizer has finite index in its neighboring vertex stabilizers. This implies that all edge and vertex stabilizers are in a single commensurability class. Because these subgroups are infinite cyclic, this class contains all nontrivial subgroups of vertex stabilizers. Therefore every nontrivial elliptic element is commensurable with all of its conjugates.

Now suppose that an element $\gamma \in G$ is commensurable with its conjugates. If $\gamma$ is hyperbolic then let $L_{\gamma}$ be its axis. For every $\delta \in G, \delta \gamma \delta^{-1}$ is commensurable with $\gamma$ and hence its axis $L_{\delta \gamma \delta^{-1}}$ is equal to $L_{\gamma}$. But $L_{\delta \gamma \delta^{-1}}=\delta L_{\gamma}$, and so we have shown that $L_{\gamma}$ is $G$-invariant. Hence $L_{\gamma}$ contains any minimal subtree.

These remarks imply that if a minimal subtree of a generalized BaumslagSolitar tree is not contained in a line, then the nontrivial elliptic elements are exactly those elements which are commensurable with all of their conjugates. Thus, $X$ and $Y$ define the same partition of $G$ into elliptic and hyperbolic elements. Then, as all vertex stabilizers have property (E) (by finite generation), 
Corollary 4.3 implies that the two trees are related by a deformation. The other conclusions follow from Theorems 4.2 and 6.1.

\section{Geometric Rigidity}

In this section we examine the preceding results from the coarse geometric point of view. We find that deformation-equivalence of $G$-trees can be characterized geometrically, as stated in condition (c) of Theorem 1.1. This result, combined with Theorem 1.2, yields the quasi-isometric rigidity theorem given in Corollary 1.5 .

Definition 7.1 A map $f: X \rightarrow Y$ between metric spaces $X$ and $Y$ is a quasiisometry if there exist constants $K \geqslant 1, C \geqslant 0$ such that for every $x, x^{\prime} \in X$,

$$
K^{-1} d\left(x, x^{\prime}\right)-C \leqslant d\left(f(x), f\left(x^{\prime}\right)\right) \leqslant K d\left(x, x^{\prime}\right)+C,
$$

and if $d(y, f(X))<C$ for every $y \in Y$.

If $X$ and $Y$ admit $G$-actions by isometries then a map $f: X \rightarrow Y$ is $L-$ equivariant if for every $x \in X$ and $\gamma \in G, d(f(\gamma x), \gamma f(x))<L$. If $f$ is $L$-equivariant for some $L$ then we say that $f$ is coarsely equivariant.

If $f: X \rightarrow Y$ is a quasi-isometry then an $M$-quasi-inverse is a quasi-isometry $g: Y \rightarrow X$ such that $d(x, g(f(x)))<M$ and $d(y, f(g(y)))<M$ for every $x \in X$ and $y \in Y$.

Lemma 7.2 Let $f: X \rightarrow Y$ be a $(K, C)$-quasi-isometry. Then there exists a $(K, 3 K C)$-quasi-isometry $g: Y \rightarrow X$ which is a $2 K C$-quasi-inverse of $f$. If $X$ and $Y$ admit $G$-actions by isometries and $f$ is $L$-equivariant, then any $M$-quasi-inverse of $f$ is $K(2 M+C+L)$-equivariant.

Proof We define $g$ by choosing $g(y)$ to be any point of $X$ with $d(f(g(y)), y)<$ $C$. It is straightforward to check that $g$ is a $(K, 3 K C)$-quasi-isometry, and a $2 K C$-quasi-inverse of $f$. Next let $g$ be any $M$-quasi-inverse to $f$, and take $\gamma \in G$ and $y \in Y$. Then

$$
\begin{aligned}
& K^{-1} d(g(\gamma y), \gamma g(y))-C \leqslant d(f(g(\gamma y)), f(\gamma g(y))) \\
& \leqslant d(f(g(\gamma y)), \gamma y)+d(\gamma y, \gamma f(g(y)))+d(\gamma f(g(y)), f(\gamma g(y))) .
\end{aligned}
$$

The middle term on the second line is equal to $d(y, f(g(y)))$ and so the second line is bounded by $2 M+L$. Hence $d(g(\gamma y), \gamma g(y)) \leqslant K(2 M+C+L)$ as desired. 
Theorem 4.2 can now be extended as follows, completing the proof of Theorem 1.1 .

Theorem 7.3 Let $G$ be a group, and let $X$ and $Y$ be cocompact $G$-trees. The following conditions are equivalent.

(a) $X$ and $Y$ have the same elliptic subgroups.

(b) There exists an elementary deformation $X \rightarrow Y$.

(c) There exists a coarsely equivariant quasi-isometry $\phi: X \rightarrow Y$.

Note in particular the implication $(\mathrm{c}) \Rightarrow(\mathrm{b})$. It transforms a coarse, approximate relationship between $G$-trees into a combinatorially precise one. We also remark that the implications $(\mathrm{b}) \Rightarrow(\mathrm{c})$ and $(\mathrm{c}) \Rightarrow(\mathrm{a})$ do not require cocompactness.

Proof The implication $(\mathrm{a}) \Rightarrow(\mathrm{b})$ is given by Theorem 4.2 . For $(\mathrm{b}) \Rightarrow(\mathrm{c})$, we claim that a collapse move is itself an equivariant quasi-isometry. Then Lemma 7.2 implies that the relation defined by condition (c) is an equivalence relation. Since the relation of condition (b) is generated by collapse moves, and the implication (b) $\Rightarrow$ (c) holds for these moves, it must hold in general.

To prove the claim, we show that a collapse move is a $(3,2 / 3)$-quasi-isometry. The essential point is that if $e$ can be collapsed, then the connected components of $G e$ have diameter at most 2. Then, if $\left(e_{1}, \ldots, e_{d}\right)$ is a geodesic of length $d$, then at most $(2 / 3)(d+1)$ of its geometric edges are in $G\{e, \bar{e}\}$. Thus the distance $d^{\prime}$ between the endpoints after the collapse satisfies

$$
(1 / 3)(d-2) \leqslant d^{\prime} \leqslant d .
$$

Now consider a collapsible edge $e$ (with $G_{e}=G_{\partial_{0} e}$ ) and a translate $\gamma e$ which is adjacent to $e$. The endpoints of $e$ are in different $G$-orbits, so either $\partial_{0} e=\partial_{0} \gamma e$ or $\partial_{1} e=\partial_{1} \gamma e$. In the former case we have that $\gamma \in G_{\partial_{0} e}-G_{e}$, a contradiction. Hence the latter holds. It follows that every edge in the connected component of $G e$ containing $e$ is incident to $\partial_{1} e$. Hence this component has diameter at most 2 .

For $(\mathrm{c}) \Rightarrow($ a) we will establish the fact that if $f: X \rightarrow Y$ is any coarsely equivariant map of $G$-trees, then every vertex stabilizer of $X$ fixes a vertex of $Y$. We then apply this fact both to $\phi$ and to a quasi-inverse $\psi: Y \rightarrow X$, which exists and is coarsely equivariant by Lemma 7.2. Then $X$ and $Y$ have the same elliptic subgroups. 
Suppose $f: X \rightarrow Y$ is $L$-equivariant. If $\gamma \in G_{x}$ for some $x \in V(X)$, then $d(\phi(x), \gamma \phi(x))=d(\phi(\gamma x), \gamma \phi(x))<L$. Thus $T$, the subtree spanned by $G_{x} \phi(x)$, is a bounded subtree of $Y$. Now every element $\gamma$ of $G_{x}$ has a fixed point in $Y$, since if it had positive translation length then the subset $\left\{\gamma^{i} \phi(x)\right\}_{i>0} \subseteq T$ would be unbounded. Note also that a fixed point of $\gamma$ lies on the path $[\phi(x), \gamma \phi(x)] \subseteq T$ and so every element of $G_{x}$ has a fixed point in $T$.

By Proposition 2.6 either $G_{x}$ has a fixed point in $Y$ or there is an end $\varepsilon \in \partial Y$ fixed by $G_{x}$. Suppose the latter holds. Let $y$ and $y^{\prime}$ be vertices of $T$. The subtree spanned by $y, y^{\prime}$, and $\varepsilon$ meets $T$ in a subtree containing $\left[y, y^{\prime}\right]$, so the intersection $[y, \varepsilon) \cap\left[y^{\prime}, \varepsilon\right) \cap T$ is nonempty. As $T$ is bounded, there is a nearest vertex $z \in[y, \varepsilon) \cap\left[y^{\prime}, \varepsilon\right) \cap T$ to $\varepsilon$, which is also the nearest vertex of $[y, \varepsilon) \cap T$ to $\varepsilon$. Fixing $y$, this shows that $z \in\left[y^{\prime}, \varepsilon\right)$ for every $y^{\prime} \in T$. Therefore $G_{x}$ fixes $z$ as each of its elements fixes both $\varepsilon$ and some vertex of $T$.

Proposition 7.4 Let $G$ be a group and $X$ a proper $G$-tree. Let $\phi: V(X) \rightarrow$ $V(X)$ be an $L$-equivariant map. Then $\operatorname{dist}(\phi, \mathrm{id})<L / 2$. In particular, if $\phi$ is equivariant then $\phi=\mathrm{id}$.

Proof Let $x$ be a vertex of $X$. We wish to show that $d(x, \phi(x))<L / 2$. For each $\gamma \in G_{x}$ we have that $d(\phi(x), \gamma \phi(x))=d(\phi(\gamma x), \gamma \phi(x))<L$. Let $m_{\gamma}$ be the vertex defined by

$$
m_{\gamma}=[x, \phi(x)] \cap[\phi(x), \gamma \phi(x)] \cap[\gamma \phi(x), x] .
$$

That is, $m_{\gamma}$ is the midpoint of the tripod spanned by $x, \phi(x)$, and $\gamma \phi(x)$. Note that $\gamma$ fixes the segment $\left[x, m_{\gamma}\right] \subseteq[x, \phi(x)]$. Suppose that $m_{\gamma} \neq x$ for every $\gamma \in G_{x}$. Then $G_{x}$ fixes an interior point of $[x, \phi(x)]$. However, as $X$ is proper, the fixed point set of $G_{x}$ is $\{x\}$, and therefore $m_{\gamma}=x$ for some $\gamma \in G_{x}$. Then $x$ is the midpoint of $[\phi(x), \gamma \phi(x)]$, a path of length less than $L$.

The following result is Corollary 1.5 of the Introduction.

Theorem 7.5 Let $G$ be a group. Let $X$ and $Y$ be reduced cocompact $G-$ trees, with $X$ strongly slide-free. If $\phi: X \rightarrow Y$ is a coarsely equivariant quasiisometry, then there is a unique equivariant isometry from $X$ to $Y$, and it has finite distance from $\phi$.

Proof The first conclusion follows directly from Theorems 7.3 and 5.17. The second conclusion follows from Proposition 7.4. 


\section{References}

[1] Hyman Bass, Covering theory for graphs of groups, J. Pure Appl. Algebra 89 (1993) 3-47

[2] Hyman Bass, Renfang Jiang, Automorphism groups of tree actions and of graphs of groups, J. Pure Appl. Algebra 112 (1996) 109-155

[3] Hyman Bass, Ravi Kulkarni, Uniform tree lattices, J. Amer. Math. Soc. 3 (1990) 843-902

[4] Hyman Bass, Alexander Lubotzky, Rigidity of group actions on locally finite trees, Proc. London Math. Soc. 69 (1994) 541-575

[5] Mladen Bestvina, Mark Feighn, Bounding the complexity of simplicial group actions on trees, Invent. Math. 103 (1991) 449-469

[6] I M Chiswell, The Grushko-Neumann theorem, Proc. London Math. Soc. 33 (1976) 385-400

[7] Marc Culler, John W Morgan, Group actions on $\mathbb{R}$-trees, Proc. London Math. Soc. 55 (1987) 571-604

[8] M J Dunwoody, Folding sequences, from: "The Epstein birthday schrift", Geometry and Topology Monographs, 1 (1998) 139-158

[9] Max Forester, On uniqueness of JSJ decompositions of finitely generated groups, preprint (2001) arXiv:math.GR/0110176

[10] Frank Herrlich, Graphs of groups with isomorphic fundamental group, Arch. Math. 51 (1988) 232-237

[11] A G Kurosh, The theory of groups, Chelsea Publishing Co., New York (1960)

[12] Lee Mosher, Michah Sageev, Kevin Whyte, Quasi-actions on trees, Research announcement, preprint (2000) arXiv:math.GR/0005210

[13] Jean-Pierre Serre, Trees, Springer-Verlag (1980)

[14] John R Stallings, Foldings of $G$-trees, from: "Arboreal group theory (Berkeley, CA, 1988)", Springer, New York (1991) 355-368

[15] J Tits, Sur le groupe des automorphismes d'un arbre, from: "Essays on Topology and Related Topics: Memoires dédiés à Georges de Rham", (A Haefliger, R Narasimhan, editors), Springer (1970) 188-211 